\title{
A GOAL-ORIENTED ADAPTIVE FINITE ELEMENT METHOD WITH CONVERGENCE RATES*
}

\author{
MARIO S. MOMMER ${ }^{\dagger}$ AND ROB STEVENSON $\ddagger$
}

\begin{abstract}
An adaptive finite element method is analyzed for approximating functionals of the solution of symmetric elliptic second order boundary value problems. We show that the method converges and derive a favorable upper bound for its convergence rate and computational complexity. We illustrate our theoretical findings with numerical results.
\end{abstract}

Key words. adaptive finite element method, convergence rates, computational complexity, quantity of interest, a posteriori error estimators

AMS subject classifications. 65N30, 65N50, 65N15, 65Y20, 41A25

DOI. $10.1137 / 060675666$

1. Introduction. Adaptive finite element methods (AFEMs) have become a standard tool for the numerical solution of partial differential equations. Although used successfully for more than 25 years, in more than one space dimension, even for the most simple case of symmetric elliptic equations of second order $a(u, v)=f(v)$ $(\forall v)$, their convergence was not demonstrated before the works of Dörfler [Dör96] and Morin, Nochetto, and Siebert [MNS00]. Convergence alone, however, does not show that the use of an AFEM for a solution that has singularities improves upon, or even competes with, that of a nonadaptive FEM. Recently, after the derivation of such a result by Binev, Dahmen, and DeVore [BDD04] for an AFEM extended with a so-called coarsening routine, in [Ste07] it was shown that standard AFEMs converge with the best possible rate in linear complexity.

The aforementioned works all deal with AFEMs in which the error is measured in the energy norm $\|\cdot\|_{E}:=a(\cdot, \cdot)^{\frac{1}{2}}$. In many applications, however, one is not so much interested in the solution $u$ as a whole, but rather in a (linear) functional $g(u)$ of the solution, often being referred to as a quantity of interest. With $u_{\tau}$ denoting the finite element approximation of $u$ with respect to a partition $\tau$, from $\mid g(u)-$ $g\left(u_{\tau}\right) \mid \leq\|g\|_{E^{\prime}}\left\|u-u_{\tau}\right\|_{E}$, obviously it follows that convergence of $u_{\tau}$ towards $u$ with respect to $\|\cdot\|_{E}$ implies that of $g\left(u_{\tau}\right)$ towards $g(u)$ with at least the same rate. It is, however, generally observed that with adaptive methods especially designed for the approximation of this quantity of interest, known as goal-oriented adaptive methods, convergence of $g\left(u_{\tau}\right)$ towards $g(u)$ takes place at a higher rate. Examples of such methods can be found in the monographs [AO00, BR03, BS01], and in references cited therein. So far these goal-oriented adaptive methods are usually not proven to converge. An exception is the method from [DKV06], however, in which adaptivity is purely driven by energy norm minimalization of the error in the dual problem $a(v, z)=$ $g(v)(\forall v)$. Another exception is the goal-oriented method from [MvSST06], which is

*Received by the editors November 22, 2006; accepted for publication (in revised form) October 16, 2008; published electronically February 6, 2009. This work was supported by the Netherlands Organization for Scientific Research and by the European Community's Human Potential Programme under contract HPRN-CT-2002-00286.

http://www.siam.org/journals/sinum/47-2/67566.html

${ }^{\dagger}$ Interdisciplinary Center for Scientific Computing (IWR), Universität Heidelberg, Im Neuenheimer Feld 368, 69120 Heidelberg, Germany (mario.mommer@iwr.uni-heidelberg.de).

${ }^{\ddagger}$ Korteweg-de Vries Institute for Mathematics, University of Amsterdam, Plantage Muidergracht 24, 1018 TV Amsterdam, The Netherlands (R.P.Stevenson@uva.nl). 
proven to converge with a rate equal to what we will demonstrate (for piecewise linears), where in [MvSST06] the strong assumption $u, z \in C^{3}(\bar{\Omega})$ was made.

The starting point of our method is the well-known upper bound

$$
\left|g(u)-g\left(u_{\tau}\right)\right|=\left|a\left(u-u_{\tau}, z-z_{\tau}\right)\right| \leq\left\|u-u_{\tau}\right\|_{E}\left\|z-z_{\tau}\right\|_{E},
$$

where $z_{\tau}$ is the finite element approximation with respect to $\tau$ of $z$. Having available an AFEM that is convergent with respect to the energy norm, in view of (1.1) an obvious approach would be to use it for finding partitions $\tau_{p}$ and $\tau_{d}$ such that the corresponding finite element approximations $u_{\tau_{p}}$ and $z_{\tau_{d}}$ have, say, both energy norm errors less than $\sqrt{\varepsilon}$. Indeed, then the product of the errors in primal and dual finite element approximations with respect to the smallest common refinement of $\tau_{p}$ and $\tau_{d}$-and thus the error in the approximation of the quantity of interest-is less than $\varepsilon$. This approach, however, would not benefit from the situation in which, quantitatively or qualitatively, either primal or dual solution is easier to approximate by finite element functions.

The alternative method we propose here works, in essence, as follows. On the $k$ th iteration, we start from a partition $\tau_{k}$ and compute on it the solutions of the primal and dual problems. To advance the iteration, this partition is refined in such a way that the product $\left\|u-u_{\tau}\right\|_{E}\left\|z-z_{\tau}\right\|_{E}$ is reduced by a constant factor. To achieve this, we consider the effort needed to reduce each of $\left\|u-u_{\tau}\right\|_{E}$ and $\left\|z-z_{\tau}\right\|_{E}$ by the same constant factor, which we do by separately computing suitable refinement sets. The smallest of these sets is then applied to $\tau_{k}$ to obtain $\tau_{k+1}$.

We can show that this method is convergent. In particular, we prove that if, for whatever $s, t>0$, the solutions of the primal and dual problems can be approximated in energy norm to any accuracy $\delta>0$ from partitions of cardinality $\mathcal{O}\left(\delta^{-1 / s}\right)$ or $\mathcal{O}\left(\delta^{-1 / t}\right)$, respectively, then given $\varepsilon>0$, our method constructs a partition of cardinality $\mathcal{O}\left(\varepsilon^{-1 /(s+t)}\right)$ such that

$$
\left|g(u)-g\left(u_{\tau}\right)\right| \leq\left\|u-u_{\tau}\right\|_{E}\left\|z-z_{\tau}\right\|_{E} \leq \varepsilon .
$$

In view of the assumptions, this order of cardinality realizing $\left\|u-u_{\tau}\right\|_{E}\left\|z-z_{\tau}\right\|_{E} \leq \varepsilon$ is optimal. Moreover, by solving the arising linear systems only inexactly, we show that the overall cost of the algorithm is of order $\mathcal{O}\left(\varepsilon^{-1 /(s+t)}\right)$.

The convergence rate $s+t$ of our goal-oriented method is thus the sum of the rates $s$ and $t$ of the best approximations in energy norm for primal and dual problems. With the approach of approximating both primal and dual problem within tolerance $\sqrt{\varepsilon}$, the rate would be $2 \min (s, t)$. Another alternative approach, namely, to solve each of the problems to an accuracy of $\varepsilon^{s /(s+t)}$ and $\varepsilon^{t /(s+t)}$, respectively, would also result in the rate $s+t$. This approach, however, is not feasible, since the values $s$ and $t$ are generally unknown. Our method converges at the rate $s+t$ without previous knowledge about the regularity of the solutions.

Concerning the value of $s$ (and similarly $t$ ), when applying finite elements of order $p$, for $s$ up to $p / n$, a rate $s$ is guaranteed when the solution has " $n s$ orders of smoothness" in $L_{\tau}(\Omega)$ for some $\tau>\left(\frac{1}{2}+s\right)^{-1}$ (instead of in $L_{2}(\Omega)$ required for nonadaptive approximation) (cf. [BDDP02]).

Our method is based on minimizing an upper bound for the error in the functional, which under certain circumstances can be crude. Actually, in all available goal-oriented adaptive methods the decision of which elements have to be refined is based on some upper bound for the error. Unlike the error in energy norm, there exists no computable two-sided bound for the error in a functional of the solution. 
This leaves open the possibility that some bounds are "usually" sharper than others. An argument against the upper bound (1.1) brought up in [BR03] is that it is based on the application of a global Cauchy-Schwarz inequality, whereas the dual weighted residual method advocated there would better respect the local information. The contribution of the current paper is that we prove a rate that is generally observed with goal-oriented methods. When applying finite element spaces of equal order at primal and dual sides, we neither expect (see Remark 5.1 for details) nor observe in our experiments that on average our bound gets increasingly more pessimistic when the iteration proceeds.

This paper is organized as follows: In section 2, we describe the model boundary value problem that we will consider. The finite element spaces and the refinement rules based on bisections of $n$-simplices are discussed in section 3. In section 4 , we give results on residual-based a posteriori energy error estimators. In section 5, we present our goal-oriented AFEM under the simplifying assumption that the righthand sides of both primal and dual problems are piecewise polynomial with respect to the initial finite element partition. We derive the aforementioned bound on the cardinality of the output partition. In section 6 , the method is extended to general right-hand sides. By replacing the exact solutions of the arising linear systems by inexact ones, it is further shown that the required number of arithmetic operations and storage locations satisfies the same favorable bound as the cardinality of the output partition. Finally, in section 7, we present numerical results obtained with the method. To apply our approach also to unbounded functionals, here we recall the use of extraction functionals, an approach introduced in [BS01].

In this paper, by $C \lesssim D$ we will mean that $C$ can be bounded by a multiple of $D$, independently of parameters upon which $C$ and $D$ may depend. Similarly, $C \gtrsim D$ is defined as $D \lesssim C$, and $C \approx D$ as $C \lesssim D$ and $C \gtrsim D$.

2. The model problem. Let $\Omega \subset \mathbb{R}^{n}$ be a polygonal domain. We consider the following model boundary value problem in variational form: Given $f \in H^{-1}(\Omega)$, find $u \in H_{0}^{1}(\Omega)$ such that

$$
a(u, v):=\int_{\Omega} \boldsymbol{A} \nabla u \cdot \nabla v=f(v) \quad\left(v \in H_{0}^{1}(\Omega)\right),
$$

where $\boldsymbol{A} \in L_{\infty}(\Omega)$ is a symmetric $n \times n$ matrix with $\operatorname{ess}_{\inf } \in \Omega \lambda_{\min }(\boldsymbol{A}(x))>0$. We assume that $\boldsymbol{A}$ is piecewise constant with respect to an initial finite element partition $\tau_{0}$ of $\Omega$ specified below. To keep the exposition simple, we do not attempt to derive results that hold uniformly in the size of jumps of $\rho(\boldsymbol{A})$ over element interfaces, although, under some conditions, this is likely possible; cf. [Ste05]. For $f \in L_{2}(\Omega)$, we interpret $f(v)$ as $\int_{\Omega} f v$.

Given some $g \in H^{-1}(\Omega)$, we will be interested in $g(u)$. With $z \in H_{0}^{1}(\Omega)$ we will denote the solution of the dual problem

$$
a(v, z)=g(v) \quad\left(v \in H_{0}^{1}(\Omega)\right) .
$$

We set the energy norm on $H_{0}^{1}(\Omega)$ and dual norm on $H^{-1}(\Omega)$ by

$$
\|v\|_{E}=a(v, v)^{\frac{1}{2}} \quad \text { and } \quad\|h\|_{E^{\prime}}=\sup _{0 \neq v \in H_{0}^{1}(\Omega)} \frac{|h(v)|}{\|v\|_{E}},
$$

respectively. 
3. Finite element spaces. Given an essentially disjoint subdivision $\tau$ of $\bar{\Omega}$ into (closed) $n$-simplices, called a partition, we will search approximations for $u$ and $z$ from the finite element space

$$
\mathbb{V}_{\tau}:=H_{0}^{1}(\Omega) \cap \prod_{T \in \tau} P_{p}(T),
$$

where $0<p \in \mathbb{N}$ is some fixed constant. For approximating the functionals $f$ and $g$, we will make use of spaces

$$
\mathbb{V}_{\tau}^{*}:=\prod_{T \in \tau} P_{p-1}(T)
$$

Although it is not a finite element space in the usual sense, we also use

$$
\mathbb{W}_{\tau}^{*}:=\prod_{T \in \tau}\left\{\boldsymbol{h} \in H(\operatorname{div} ; T): \llbracket \boldsymbol{h} \cdot \mathbf{n} \rrbracket_{\partial T} \in L_{2}(\partial T)\right\},
$$

with $\mathbf{n}$ being a unit vector normal to $\partial T$, and $\llbracket \rrbracket_{\partial T}$ denoting the jump of its argument over $\partial T$ in the direction of $\mathbf{n}$, defined to be zero on $\partial \Omega$. Obviously, $\left[\mathbb{V}_{\tau}^{*}\right]^{n} \subset \mathbb{W}_{\tau}^{*}$.

Below, we specify the type of (nested) partitions we will consider, and we recall some results from [Ste08], generalizing upon known results for newest vertex bisection in two dimensions.

For $0 \leq k \leq n-1$, a (closed) simplex spanned by $k+1$ vertices of an $n$-simplex $T$ is called a hyperface of $T$. For $k=n-1$, it will be called a true hyperface. A partition $\tau$ is called conforming when the intersection of any two different $T, T^{\prime} \in \tau$ is either empty or a hyperface of both simplices. Different simplices $T, T^{\prime}$ that share a true hyperface will be called neighbors. (Actually, when $\Omega \neq \operatorname{int}(\bar{\Omega})$, the above definition of a conforming partition can be unnecessarily restrictive. We refer to [Ste08] for a discussion of this matter.)

Simplices will be refined by means of bisection. In order to guarantee uniform shape regularity of all descendants, a proper cyclic choice of the refinement edges should be made. To that end, given $\left\{x_{0}, \ldots x_{n}\right\} \subset \mathbb{R}^{n}$, not on a joint $(n-1)$ dimensional hyperplane, we distinguish between $n(n+1)$ ! tagged simplices given by all possible ordered sequences $\left(x_{0}, x_{1}, \ldots, x_{n}\right)_{\gamma}$ and types $\gamma \in\{0, \ldots, n-1\}$. Given a tagged simplex $T=\left(x_{0}, x_{1}, \ldots, x_{n}\right)_{\gamma}$, its children are the tagged simplices

$$
\left(x_{0}, \frac{x_{0}+x_{n}}{2}, x_{1}, \ldots, x_{\gamma}, x_{\gamma+1}, \ldots, x_{n-1}\right)_{(\gamma+1) \bmod n}
$$

and

$$
\left(x_{n}, \frac{x_{0}+x_{n}}{2}, x_{1}, \ldots, x_{\gamma}, x_{n-1}, \ldots, x_{\gamma+1}\right)_{(\gamma+1) \bmod n},
$$

where the sequences $\left(x_{\gamma+1}, \ldots, x_{n-1}\right)$ and $\left(x_{1}, \ldots, x_{\gamma}\right)$ should be read as being void for $\gamma=n-1$ and $\gamma=0$, respectively. So these children are defined by bisecting the edge $\overline{x_{0} x_{n}}$ of $T$-i.e., by connecting its midpoint with the other vertices $x_{1}, \ldots, x_{n-1}-$ by an appropriate ordering of their vertices and by having type $(\gamma+1) \bmod n$. See Figure 3.1 for an illustration. This bisection process was introduced in [Tra97] and, using different notation, in [Mau95]. The edge $\overline{x_{0} x_{n}}$ is called the refinement edge of $T$. In the $n=2$ case, the vertex opposite this edge is known as the newest vertex.

Corresponding to a tagged simplex $T=\left(x_{0}, \ldots, x_{n}\right)_{\gamma}$, we set

$$
T_{\mathrm{R}}=\left(x_{n}, x_{1}, \ldots, x_{\gamma}, x_{n-1}, \ldots, x_{\gamma+1}, x_{0}\right)_{\gamma},
$$




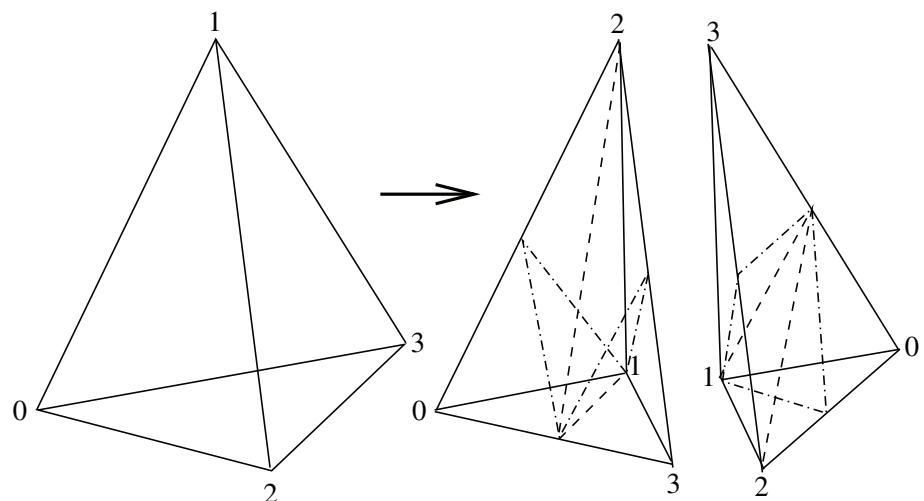

FIG. 3.1. Bisection of a tagged tetrahedron of type 0 with the next two-level cuts indicated.
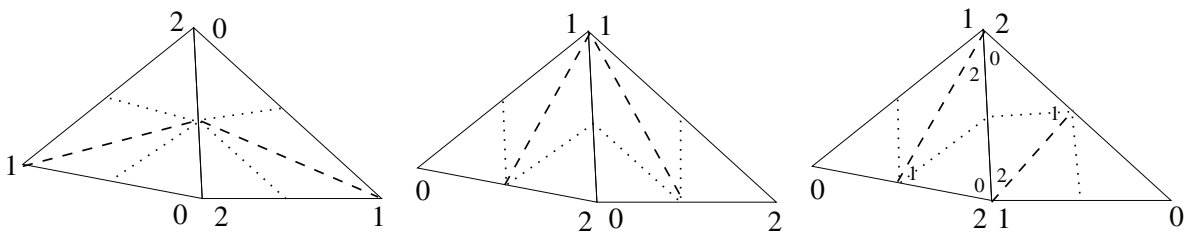

FIG. 3.2. Matching neighbors for $n=2$, and their level 1 and 2 descendants. The neighbors in the rightmost picture are not reflected neighbors, but the pair of their neighboring children are.

which is the tagged simplex that has the same set of children as $T$, and in this sense is equal to $T$. So actually we distinguish between $\frac{1}{2} n(n+1)$ ! tagged simplices.

Given a fixed conforming initial partition $\tau_{0}$ of tagged simplices of some fixed type $\gamma$,

we will exclusively consider partitions that can be created from $\tau_{0}$ by recurrent bisections of tagged simplices, in short, descendants of $\tau_{0}$.

Simplices that can be created in this way are uniformly shape regular, dependent only on $\tau_{0}$ and $n$. For the case that $\Omega$ might have slits, we assume that

$\partial \Omega$ is the union of true hyperfaces of $T \in \tau_{0}$.

We will assume that the simplices from $\tau_{0}$ are tagged in such a way that any two neighbors $T=\left(x_{0}, \ldots, x_{n}\right)_{\gamma}, T^{\prime}=\left(x_{0}^{\prime}, \ldots, x_{n}^{\prime}\right)_{\gamma}$ from $P_{0}$ match in the sense that if $\overline{x_{0} x_{n}}$ or $\overline{x_{0}^{\prime} x_{n}^{\prime}}$ is on $T \cap T^{\prime}$, then either $T$ and $T^{\prime}$ are reflected neighbors, meaning that the ordered sequence of vertices of either $T$ or $T_{\mathrm{R}}$ coincides with that of $T^{\prime}$ on all but one position, or the pair of neighboring children of $T$ and $T^{\prime}$ are reflected neighbors. See Figure 3.2 for an illustration. It is known (see [BDD04] and the references therein) that for any conforming partition into triangles there exists a local numbering of the vertices so that the matching condition is satisfied. We do not now whether the corresponding statement holds in more space dimensions. Yet we showed that any conforming partition of $n$-simplices can be refined, inflating the number of simplices by not more than an absolute constant factor, into a conforming partition $\tau_{0}$ that allows a local numbering of the vertices so that the matching condition is satisfied. 
For applying a posteriori error estimators, we will require that the partitions $\tau$ underlying the approximation spaces be conforming. So in the following

$$
\tau, \tau^{\prime}, \hat{\tau} \text {, etc., will always denote conforming partitions. }
$$

Bisecting one or more simplices in a conforming partition $\tau$ generally results in a nonconforming partition $\varrho$. Conformity has to be restored by (recursively) bisecting any simplex $T \in \varrho$ that contains a vertex $v$ of a $T^{\prime} \in \varrho$ that does not coincide with any vertex of $T$ (such a $v$ is called a hanging vertex). This process, called completion, results in the smallest conforming refinement of $\varrho$.

Our adaptive method will be of the following form:

for $j:=1$ to $M$

do create some, possibly nonconforming refinement $\varrho_{j}$ of $\tau_{j-1}$

complete $\varrho_{j}$ to its smallest conforming refinement $\tau_{j}$

endfor

As we will see, we will be able to bound $\sum_{j=1}^{M} \# \varrho_{j}-\# \tau_{j-1}$. Because of the additional bisections made in the completion steps, however, generally $\# \tau_{M}-\# \tau_{0}$ will be larger. The following crucial result, which relies on the matching condition in the initial partition, shows that these additional bisections inflate the total number of simplices by at most an absolute constant factor.

Theorem 3.1 (generalizes upon [BDD04, Theorem 2.4] for $n=2$ ).

$$
\# \tau_{M}-\# \tau_{0} \lesssim \sum_{j=1}^{M} \# \varrho_{j}-\# \tau_{j-1},
$$

dependent only on $\tau_{0}$ and $n$, and in particular thus independently of $M$.

Remark 3.2. Note that this result in particular implies that any descendant $\varrho$ of $\tau_{0}$ has a conforming refinement $\tau$ with $\# \tau \lesssim \# \varrho$, dependent only on $\tau_{0}$ and $n$.

We end this section by introducing two more notations. For partitions $\tau^{\prime}, \tau$, we write $\tau^{\prime} \supseteq \tau\left(\tau^{\prime} \supset \tau\right)$ to denote that $\tau^{\prime}$ is a (proper) refinement of $\tau$. The smallest common refinement of $\tau$ and $\tau^{\prime}$ will be denoted as $\tau \cup \tau^{\prime}$.

4. A posteriori estimators for the energy error. Given a partition $\tau$, and with $u_{\tau}$ denoting the solution in $\mathbb{V}_{\tau}$ of

$$
a\left(u_{\tau}, v_{\tau}\right)=f\left(v_{\tau}\right) \quad\left(v_{\tau} \in \mathbb{V}_{\tau}\right)
$$

in this section we discuss properties of the common residual-based a posteriori error estimator for $\left\|u-u_{\tau}\right\|_{E}$. Since $a($,$) is symmetric, an analogous result will apply to$ $\left\|z-z_{\tau}\right\|_{E}$, with $z_{\tau}$ denoting the solution in $\mathbb{V}_{\tau}$ of

$$
a\left(v_{\tau}, z_{\tau}\right)=g\left(v_{\tau}\right) \quad\left(v_{\tau} \in \mathbb{V}_{\tau}\right) .
$$

By formally viewing $H_{0}^{1}(\Omega)$ as $\mathbb{V}_{\tau}$ corresponding to the infinitely uniformly refined partition $\tau=\infty$, at some places we interpreted results derived for $u_{\tau}$ to hold for the solution $u$ of (2.1) by substituting $\tau=\infty$.

For developing an AFEM that reduces the error in each iteration, it will be necessary to approximate the right-hand side by discrete functions. Loosely speaking, in [MNS00] the error in this approximation is called data oscillation. Being on a partition $\tau$, it will be allowed to use functions from $\mathbb{V}_{\tau}^{*}+\operatorname{div}\left[\mathbb{V}_{\tau}^{*}\right]^{n}$, where $\operatorname{div}:=$ $(-\nabla)^{\prime}: L_{2}(\Omega)^{n} \rightarrow H^{-1}(\Omega)$. Depending on the right-hand side at hand, it might be 
more convenient to approximate it by functions from $\mathbb{V}_{\tau}^{*}$ or from $\operatorname{div}\left[\mathbb{V}_{\tau}^{*}\right]^{n}$, or by a combination of these. In view of this, we will write

$$
f=f^{1}+\operatorname{div} \boldsymbol{f}^{2}
$$

where $f^{1} \in H^{-1}(\Omega)$ and $f^{2} \in L_{2}(\Omega)^{n}$ are going to be approximated by functions from $\mathbb{V}_{\tau}^{*}$ or from $\operatorname{div}\left[\mathbb{V}_{\tau}^{*}\right]^{n}$, respectively. Similarly, we write $g=g^{1}+\operatorname{div} \boldsymbol{g}^{2}$.

Remark 4.1. Obviously, any $f \in H^{-1}(\Omega)$ can be written in the above form with vanishing $\boldsymbol{f}^{2}$. On the other hand, by taking $\boldsymbol{f}^{2}=-\nabla w$ with $w \in H_{0}^{1}(\Omega)$ being the solution of $\int_{\Omega} \nabla w \cdot \nabla v=f(v)\left(v \in H_{0}^{1}(\Omega)\right)$, we see that we can equally well consider a vanishing $f^{1}$.

For $\bar{u}_{\tau} \in \mathbb{V}_{\tau}, \bar{f}^{1} \in L_{2}(\Omega)$, and $\overline{\boldsymbol{f}}^{2} \in \mathbb{W}_{\tau}^{*}$ (see (3.1)), where we have in mind approximations to $u_{\tau}, f^{1}$, and $\boldsymbol{f}^{2}$, respectively, and $T \in \tau$, we set the local error indicator

$$
\begin{aligned}
\eta_{T}\left(\bar{f}^{1}, \overline{\boldsymbol{f}}^{2}, \bar{u}_{\tau}\right):= & \operatorname{diam}(T)^{2}\left\|\bar{f}^{1}+\nabla \cdot\left[\boldsymbol{A} \nabla \bar{u}_{\tau}+\overline{\boldsymbol{f}}^{2}\right]\right\|_{L_{2}(T)}^{2} \\
& +\operatorname{diam}(T)\left\|\llbracket\left[\boldsymbol{A} \nabla \bar{u}_{\tau}+\overline{\boldsymbol{f}}^{2}\right] \cdot \mathbf{n} \rrbracket \partial T\right\|_{L_{2}(\partial T)}^{2} .
\end{aligned}
$$

Note that the first term is the weighted local residual of the equation in strong form. We set the energy error estimator

$$
\mathcal{E}\left(\tau, \bar{f}^{1}, \overline{\boldsymbol{f}}^{2}, \bar{u}_{\tau}\right):=\left[\sum_{T \in \tau} \eta_{T}\left(\bar{f}^{1}, \overline{\boldsymbol{f}}^{2}, \bar{u}_{\tau}\right)\right]^{\frac{1}{2}} .
$$

The following Proposition 4.2 is a generalization of [Ste07, Theorem 4.1] valid for $\boldsymbol{A}=\mathrm{Id}, \boldsymbol{f}^{2}=0$, and polynomial degree $p=1$. This result in turn was a generalization of [BMN02, Lemma 5.1, eq. (5.4)] (see also [Ver96]) in the sense that instead of $\left\|u-u_{\tau}\right\|_{E}$, the difference $\left\|u_{\tau^{\prime}}-u_{\tau}\right\|_{E}$ for any $\tau^{\prime} \supset \tau$ is estimated. Proposition 4.2 tells us that this difference can be bounded from above by the square root of the sum of the local error indicators corresponding to those simplices from $\tau$ that either are not in $\tau^{\prime}$ since they were refined or have nonempty intersection with such simplices. By taking $\tau^{\prime}=\infty$, this result yields the known bound for $\left\|u-u_{\tau}\right\|_{E}$.

Proposition 4.2. Let $\tau^{\prime} \supset \tau$ be partitions, and let $f^{1} \in L_{2}(\Omega), f^{2} \in \mathbb{W}_{\tau}^{*}$, and

$$
G=G\left(\tau, \tau^{\prime}\right):=\left\{T \in \tau: T \cap \tilde{T} \neq \emptyset \text { for some } \tilde{T} \in \tau, \tilde{T} \notin \tau^{\prime}\right\} .
$$

Then we have

$$
\left\|u_{\tau^{\prime}}-u_{\tau}\right\|_{E} \leq C_{1}\left[\sum_{T \in G} \eta_{T}\left(f^{1}, \boldsymbol{f}^{2}, u_{\tau}\right)\right]^{\frac{1}{2}}
$$

for some absolute constant $C_{1}>0$. Note that $\# G \lesssim \# \tau^{\prime}-\# \tau$.

In particular, by taking $\tau^{\prime}=\infty$, we have

$$
\left\|u-u_{\tau}\right\|_{E} \leq C_{1} \mathcal{E}\left(\tau, f^{1}, \boldsymbol{f}^{2}, u_{\tau}\right) .
$$

Proof. We have $\left\|u_{\tau^{\prime}}-u_{\tau}\right\|_{E}=\sup _{0 \neq v_{\tau^{\prime}} \in \mathbb{V}_{\tau^{\prime}}} \frac{\left|a\left(u_{\tau^{\prime}}-u_{\tau}, v_{\tau^{\prime}}\right)\right|}{\left\|v_{\tau^{\prime}}\right\|_{E}}$. For any $v_{\tau^{\prime}} \in \mathbb{V}_{\tau^{\prime}}$, $v_{\tau} \in \mathbb{V}_{\tau}$, we have

$$
\begin{aligned}
a\left(u_{\tau^{\prime}}\right. & \left.-u_{\tau}, v_{\tau^{\prime}}\right)=a\left(u_{\tau^{\prime}}-u_{\tau}, v_{\tau^{\prime}}-v_{\tau}\right) \\
& =\sum_{T} \int_{T} f^{1}\left(v_{\tau^{\prime}}-v_{\tau}\right)-\boldsymbol{f}^{2} \cdot \nabla\left(v_{\tau^{\prime}}-v_{\tau}\right)-\boldsymbol{A} \nabla u_{\tau^{\prime}} \cdot \nabla\left(v_{\tau^{\prime}}-v_{\tau}\right) \\
& =\sum_{T}\left\{\left(f^{1}+\nabla \cdot\left[\boldsymbol{A} \nabla u_{\tau}+\boldsymbol{f}^{2}\right]\right)\left(v_{\tau^{\prime}}-v_{\tau}\right)-\int_{\partial T}\left[\boldsymbol{A} \nabla u_{\tau}+\boldsymbol{f}^{2}\right] \cdot \mathbf{n}\left(v_{\tau^{\prime}}-v_{\tau}\right)\right\},
\end{aligned}
$$


where the last line follows by integration by parts. By taking $v_{\tau}$ to be a suitable local quasi-interpolant of $v_{\tau^{\prime}}$ as in [Ste07] (for $p>1$, one may consult [KS08]) or, alternatively, a Clément-type interpolator, and applying a Cauchy-Schwarz inequality, one completes the proof.

Remark 4.3. For the lowest order elements, i.e., $p=1$, a statement similar to Proposition 4.2 is valid with error indicators consisting of the jump terms over the interfaces only. As a consequence, along the lines that we will follow for elements of general degree $p$, for $p=1$ a cheaper goal-oriented AFEM can be developed that has similar properties. Details can be found in Appendix A of the extended preprint version [MS08] of this work.

Next we study whether the error estimator also provides a lower bound for $\| u-$ $u_{\tau} \|_{E}$ and, when $\tau^{\prime}$ is a sufficient refinement of $\tau$, for $\left\|u_{\tau^{\prime}}-u_{\tau}\right\|_{E}$. In order to derive such estimates, for the moment we further restrict the type of right-hand sides. The proof of the following proposition will be derived along the lines of the proof of [BMN02, Lemma 5.3], where the Stokes problem is considered (see also [MNS00, Lemma 4.2] for the case $p=1$ and $\boldsymbol{f}^{2}=0$ ). For convenience of the reader we include it here.

Proposition 4.4. Let $\tau \subset \tau^{\prime}$ be partitions, and let $f^{1} \in \mathbb{V}_{\tau}^{*}, f^{2} \in\left[\mathbb{V}_{\tau}^{*}\right]^{n}$, and $\bar{u}_{\tau} \in \mathbb{V}_{\tau}$.

(a) If $T \in \tau$ contains a vertex of $\tau^{\prime}$ in its interior, then

$$
\operatorname{diam}(T)^{2}\left\|f^{1}+\nabla \cdot\left[\boldsymbol{A} \nabla \bar{u}_{\tau}+\boldsymbol{f}^{2}\right]\right\|_{L_{2}(T)}^{2} \lesssim\left|u_{\tau^{\prime}}-\bar{u}_{\tau}\right|_{H^{1}(T)}^{2} .
$$

(b) If a joint true hyperface e of $T_{1}, T_{2} \in \tau$ contains a vertex of $\tau^{\prime}$ in its interior, then

$$
\begin{aligned}
\operatorname{diam}(e)\left\|\llbracket\left[\boldsymbol{A} \nabla \bar{u}_{\tau}+\boldsymbol{f}^{2}\right] \cdot \boldsymbol{n} \rrbracket_{e}\right\|_{L_{2}(e)}^{2} \lesssim\left|u_{\tau^{\prime}}-\bar{u}_{\tau}\right|_{H^{1}\left(T_{1} \cup T_{2}\right)}^{2} \\
+\sum_{i=1}^{2} \operatorname{diam}\left(T_{i}\right)^{2}\left\|f^{1}+\nabla \cdot\left[\boldsymbol{A} \nabla \bar{u}_{\tau}+\boldsymbol{f}^{2}\right]\right\|_{L_{2}\left(T_{i}\right)}^{2} .
\end{aligned}
$$

Proof. Let $\phi_{T} \in H_{0}^{1}(\Omega) \cap \prod_{T^{\prime} \in \tau^{\prime}} P_{1}\left(T^{\prime}\right)$ be the canonical nodal basis function associated to a vertex of $\tau^{\prime}$ inside $T$. Writing $R_{T}=\left.\left(f^{1}+\nabla \cdot\left[\boldsymbol{A} \nabla \bar{u}_{\tau}+\boldsymbol{f}^{2}\right]\right)\right|_{T} \in P_{d-1}(T)$, and $v_{\tau^{\prime}}=R_{T} \phi_{T} \in \mathbb{V}_{\tau^{\prime}}$, using the fact that $\operatorname{supp} v_{\tau^{\prime}} \subset T$, by integration by parts we get

$$
\begin{aligned}
\int_{T} R_{T}^{2} \lesssim \int_{T} R_{T}^{2} \phi_{T}=\int_{T} R_{T} v_{\tau^{\prime}} & =\left(f_{1}+\operatorname{div} \boldsymbol{f}^{2}\right)\left(v_{\tau^{\prime}}\right)-\int_{T} \boldsymbol{A} \nabla \bar{u}_{\tau} \cdot \nabla v_{\tau}^{\prime} \\
& =\int_{T} \boldsymbol{A} \nabla\left(u_{\tau^{\prime}}-\bar{u}_{\tau}\right) \cdot \nabla v_{\tau^{\prime}},
\end{aligned}
$$

and so by $\left|v_{\tau^{\prime}}\right|_{H^{1}(T)} \lesssim \operatorname{diam}(T)^{-1}\left\|v_{\tau^{\prime}}\right\|_{L_{2}(T)} \lesssim \operatorname{diam}(T)^{-1}\left\|R_{T}\right\|_{L_{2}(T)}$, we infer (a).

Let $\phi_{e} \in H_{0}^{1}(\Omega) \cap \prod_{T^{\prime} \in \tau^{\prime}} P_{1}\left(T^{\prime}\right)$ be the canonical nodal basis function associated to a vertex interior to $e$. Writing $J_{e}=\llbracket\left[\boldsymbol{A} \nabla \bar{u}_{\tau}+\boldsymbol{f}^{2}\right] \cdot \boldsymbol{n} \rrbracket_{e} \in P_{d-1}(e)$, let $\bar{J}_{e} \in$ $P_{d-1}\left(T_{1} \cup T_{2}\right)$ denote its extension constant in the direction normal to $e$, and let $v_{\tau^{\prime}}=\bar{J}_{e} \phi_{e} \in \mathbb{V}_{\tau^{\prime}}$. Using the fact that $\operatorname{supp} v_{\tau} \subset T_{1} \cup T_{2}$, by integration by parts we get

$$
\int_{e} J_{e}^{2} \lesssim \int_{e} J_{e}^{2} \phi_{e}=\int_{e} J_{e} v_{\tau^{\prime}}=\int_{T_{1} \cup T_{2}}\left(\boldsymbol{A} \nabla \bar{u}_{\tau}+\boldsymbol{f}^{2}\right) \cdot \nabla v_{\tau^{\prime}}+\int_{T_{1} \cup T_{2}} \nabla \cdot\left(\boldsymbol{A} \nabla \bar{u}_{\tau}+\boldsymbol{f}^{2}\right) v_{\tau^{\prime}} .
$$


From

$$
\int_{T_{1} \cup T_{2}} \boldsymbol{f}^{2} \cdot \nabla v_{\tau^{\prime}}=-\operatorname{div} \boldsymbol{f}^{2}\left(v_{\tau^{\prime}}\right)=-a\left(u_{\tau^{\prime}}, v_{\tau^{\prime}}\right)+\int_{T_{1} \cup T_{2}} f^{1} v_{\tau^{\prime}}
$$

we infer

$$
\begin{aligned}
\int_{e} J_{e}^{2} & \lesssim a\left(\bar{u}_{\tau}-u_{\tau^{\prime}}, v_{\tau^{\prime}}\right)+\int_{T_{1} \cup T_{2}}\left(f^{1}+\nabla \cdot\left(\boldsymbol{A} \nabla \bar{u}_{\tau}+\boldsymbol{f}^{2}\right)\right) v_{\tau^{\prime}} \\
& \lesssim\left[\left|\bar{u}_{\tau}-u_{\tau^{\prime}}\right|_{H^{1}\left(T_{1} \cup T_{2}\right)} \operatorname{diam}(e)^{-1}+\sum_{i=1}^{2}\left\|R_{T_{i}}\right\|_{L_{2}\left(T_{i}\right)}\right]\left\|v_{\tau^{\prime}}\right\|_{L_{2}\left(T_{1} \cup T_{2}\right)} .
\end{aligned}
$$

Using the fact that $\left\|v_{\tau^{\prime}}\right\|_{L_{2}\left(T_{1} \cup T_{2}\right)} \bar{\sim}\left\|\bar{J}_{e}\right\|_{L_{2}\left(T_{1} \cup T_{2}\right)} \approx \operatorname{diam}(e)^{\frac{1}{2}}\left\|J_{e}\right\|_{L_{2}(e)}$, we infer item (b) of the proposition. $\quad \square$

In view of this last result, we will call a (possibly nonconforming) $\varrho \supset \tau$ a full refinement with respect to $T \in \tau$ when

$T$, and its neighbors in $\tau$, as well as all true hyperfaces of $T$, all contain a vertex of $\varrho$ in their interiors.

As a direct consequence of Proposition 4.4 we have the following.

Corollary 4.5. Let $\tau$ be a partition, let $f^{1} \in \mathbb{V}_{\tau}^{*}, f^{2} \in\left[\mathbb{V}_{\tau}^{*}\right]^{n}$, and $\bar{u}_{\tau} \in \mathbb{V}_{\tau}$, and let $\tau^{\prime} \supset \tau$ be a full refinement of $\tau$ with respect to all $T$ from some $F \subset \tau$. Then

$$
c_{2}\left[\sum_{T \in F} \eta_{T}\left(f^{1}, \boldsymbol{f}^{2}, \bar{u}_{\tau}\right)\right]^{\frac{1}{2}} \leq\left\|u_{\tau^{\prime}}-\bar{u}_{\tau}\right\|_{E}
$$

for some absolute constant $c_{2}>0$. In particular, we have

$$
c_{2} \mathcal{E}\left(\tau, f^{1}, \boldsymbol{f}^{2}, \bar{u}_{\tau}\right) \leq\left\|u-\bar{u}_{\tau}\right\|_{E} .
$$

Next, we investigate the stability of the energy error estimator.

Proposition 4.6. Let $\tau$ be a partition, and let $f^{1} \in L_{2}(\Omega), f^{2} \in \mathbb{W}_{\tau}^{*}$, and $v_{\tau}, w_{\tau} \in \mathbb{V}_{\tau}$. Then

$$
c_{2}\left|\mathcal{E}\left(\tau, f^{1}, \boldsymbol{f}^{2}, v_{\tau}\right)-\mathcal{E}\left(\tau, f^{1}, \boldsymbol{f}^{2}, w_{\tau}\right)\right| \leq\left\|v_{\tau}-w_{\tau}\right\|_{E} .
$$

Proof. For $\tilde{f}^{1} \in L_{2}(\Omega), \tilde{\boldsymbol{f}}^{2} \in \mathbb{W}_{\tau}^{*}$, and $v_{\tau}, w_{\tau} \in \mathbb{V}_{\tau}$, by two applications of the triangle inequality in the form $|\|\cdot\|-\|\cdot\||^{2} \leq\|\cdot-\cdot\|^{2}$, first for vectors and then for functions, we have

$$
\left|\mathcal{E}\left(\tau, f^{1}, \boldsymbol{f}^{2}, v_{\tau}\right)-\mathcal{E}\left(\tau, \tilde{f}^{1}, \tilde{\boldsymbol{f}}^{2}, w_{\tau}\right)\right| \leq \mathcal{E}\left(\tau, f^{1}-\tilde{f}^{1}, \boldsymbol{f}^{2}-\tilde{\boldsymbol{f}}^{2}, v_{\tau}-w_{\tau}\right) .
$$

By substituting $\tilde{f}^{1}=f^{1}$ and $\tilde{\boldsymbol{f}}^{2}=\boldsymbol{f}^{2}$, and by applying (4.6) the proof is complete.

5. An idealized goal-oriented AFEM. From (2.2) and $u-u_{\tau} \perp_{a(,)} \mathbb{V}_{\tau} \ni z_{\tau}$, we have

$$
\left|g(u)-g\left(u_{\tau}\right)\right|=\left|a\left(u-u_{\tau}, z\right)\right|=\left|a\left(u-u_{\tau}, z-z_{\tau}\right)\right| \leq\left\|u-u_{\tau}\right\|_{E}\left\|z-z_{\tau}\right\|_{E} .
$$

We will develop an adaptive method for minimizing the right-hand side of this expression. 
Remark 5.1. A question that naturally arises is whether there is something to be gained from using finite elements of different orders for the dual and the primal problems. Note that the derivation of (5.1) remains valid if the dual solution is computed in a lower order space, or for that matter in any space that is a subspace of $\mathbb{V}_{\tau}$. But this will result in a larger $\left\|z-z_{\tau}\right\|_{E}$, worsening our error estimate without changing the actual error $\left|g(u)-g\left(u_{\tau}\right)\right|$.

And how about using a higher order space for the dual problem? In this case, (5.1) no longer holds. As $g(u)=f(z)$, we can approximate it by $f\left(z_{\tau}\right)$ with

$$
\left|f(z)-f\left(z_{\tau}\right)\right|=\left|a\left(u, z-z_{\tau}\right)\right|=\left|a\left(u-u_{\tau}, z-z_{\tau}\right)\right| \leq\left\|u-u_{\tau}\right\|_{E}\left\|z-z_{\tau}\right\|_{E} .
$$

Thus, as before, we obtain a worse error estimate than if we had used the same higher order space for the primal problem as well.

We conclude that with our approach there is no gain from using different orders and, accordingly, will consider here only spaces of equal order.

Up to and including Lemma 5.3, we start with discussing a method for reducing $\left\|u-u_{\tau}\right\|_{E}$ or similarly $\left\|z-z_{\tau}\right\|_{E}$ separately. For some fixed

$$
\theta \in\left(0, \frac{c_{2}}{C_{1}}\right)
$$

we will make use of the following routine to mark simplices for refinement:

$\operatorname{MARK}\left[\tau, \bar{f}^{1}, \overline{\boldsymbol{f}}^{2}, \bar{u}_{\tau}\right] \rightarrow F$

$\% \bar{f}^{1} \in L_{2}(\Omega), \bar{f}^{2} \in \mathbb{W}_{\tau}^{*}, \bar{u}_{\tau} \in \mathbb{V}_{\tau}$.

Select, in $\mathcal{O}(\# \tau)$ operations, a set $F \subset \tau$ with, up to some absolute factor, minimal cardinality such that

$$
\sum_{T \in F} \eta_{T}\left(\bar{f}^{1}, \overline{\boldsymbol{f}}^{2}, \bar{u}_{\tau}\right) \geq \theta^{2} \mathcal{E}\left(\tau, \bar{f}^{1}, \overline{\boldsymbol{f}}^{2}, \bar{u}_{\tau}\right)^{2}
$$

Remark 5.2. Selecting $F$ that satisfies (5.3) with truly minimal cardinality would require the sorting of all $\eta_{T}=\eta_{T}\left(\bar{f}^{1}, \overline{\boldsymbol{f}}^{2}, \bar{u}_{\tau}\right)$, which takes $\mathcal{O}(\# \tau \log (\# \tau))$ operations. The log-factor can be avoided by performing an approximate sorting based on binning that we recall here: With $N:=\# \tau$, we may discard all $\eta_{T} \leq$ $\left(1-\theta^{2}\right) \mathcal{E}\left(\tau, \bar{f}^{1}, \overline{\boldsymbol{f}}^{2}, \bar{u}_{\tau}\right)^{2} / N$. With $M:=\max _{T \in \tau} \eta_{T}$, and $q$ the smallest integer with $2^{-q-1} M \leq\left(1-\theta^{2}\right) \mathcal{E}\left(P^{c}, \bar{f}^{1}, \bar{f}^{2}, w_{P^{c}}\right)^{2} / N$, we store the others in $q+1$ bins depending on whether $\eta_{T}$ is in $\left[M, \frac{1}{2} M\right),\left[\frac{1}{2} M, \frac{1}{4} M\right), \ldots$, or $\left[2^{-q} M, 2^{-q-1} M\right)$. Then we build $F$ by extracting $\eta_{T}$ from the bins, starting with the first bin, moving to the second bin when the first is empty, and so on until (5.3) is satisfied. Let the resulting $F$ now contain $\eta_{T}$ from the $\ell$ th bin, but not from further bins. Then a minimal set $\tilde{F}$ that satisfies (5.3) contains all $\eta_{T}$ from the bins up to the $(\ell-1)$ th one. Since any two $\eta_{T}$ in the $\ell$ th bin differ at most by a factor of 2 , we infer that the cardinality of the contribution from the $\ell$ th bin to $F$ is at most twice as large as that to $\tilde{F}$, so that $\# F \leq 2 \# \tilde{F}$. Assuming that each evaluation of $\eta_{T}$ takes $\mathcal{O}(1)$ operations, the number of operations and storage locations required by this procedure is $\mathcal{O}(q+\# \tau)$, with $q<\log _{2}\left(M N /\left[\left(1-\theta^{2}\right) \mathcal{E}\left(\tau, \bar{f}^{1}, \bar{f}^{2}, \bar{u}_{\tau}\right)^{2}\right]\right) \leq \log _{2}\left(N /\left(1-\theta^{2}\right)\right) \lesssim \log _{2}(\# \tau)<\# \tau$. The assumption on the cost of evaluating $\eta_{T}$ is satisfied when $\bar{f}^{1} \in \mathbb{V}_{\tau}^{*}$ and $\bar{f}^{2} \in\left[\mathbb{V}_{\tau}^{*}\right]^{n}$, as will be the case in our applications.

Having a set of marked elements $F$, the next step is to apply the following: 
$\operatorname{REFINE}[\tau, F] \rightarrow \tau^{\prime}$

$\%$ Determines the smallest $\tau^{\prime} \supseteq \tau$ which is a full refinement

$\%$ with respect to all $T \in F$.

The cost of the call is $\mathcal{O}\left(\# \tau^{\prime}\right)$ operations.

Using the results on the a posteriori error estimator derived in the previous section, we have the following result.

Lemma 5.3. Let $f^{1} \in \mathbb{V}_{\tau}^{*}, \boldsymbol{f}^{2} \in\left[\mathbb{V}_{\tau}^{*}\right]^{n}$. Then for $F=\operatorname{MARK}\left[\tau, f^{1}, \boldsymbol{f}^{2}, u_{\tau}\right]$ and $\tau^{\prime} \supseteq \operatorname{REFINE}[\tau, F]$, we have

$$
\left\|u-u_{\tau^{\prime}}\right\|_{E} \leq\left[1-\frac{c_{2}^{2} \theta^{2}}{C_{1}^{2}}\right]^{\frac{1}{2}}\left\|u-u_{\tau}\right\|_{E} .
$$

Furthermore

$$
\# F \lesssim \# \hat{\tau}-\# \tau_{0}
$$

for any partition $\hat{\tau}$ for which

$$
\left\|u-u_{\hat{\tau}}\right\|_{E} \leq\left[1-\frac{C_{1}^{2} \theta^{2}}{c_{2}^{2}}\right]^{\frac{1}{2}}\left\|u-u_{\tau}\right\|_{E} .
$$

Proof. Since this is a key result, for convenience of the reader we recall the arguments from [Ste07].

From

$$
\left\|u-u_{\tau}\right\|_{E}^{2}=\left\|u-u_{\tau^{\prime}}\right\|_{E}^{2}+\left\|u_{\tau^{\prime}}-u_{\tau}\right\|_{E}^{2}
$$

and, by (4.5), (5.3), and (4.4),

$$
\left\|u_{\tau^{\prime}}-u_{\tau}\right\|_{E} \geq c_{2} \theta \mathcal{E}\left(\tau, f^{1}, f^{2}, u_{\tau}\right) \geq \frac{c_{2} \theta}{C_{1}}\left\|u-u_{\tau}\right\|_{E},
$$

we conclude (5.4).

With $\hat{\tau}$ being a partition as in the statement of the theorem, let $\breve{\tau}=\tau \cup \hat{\tau}$. Then, as $\tau$ and $\hat{\tau}$, the partition $\breve{\tau}$ is a conforming descendant of $\tau_{0},\left\|u-u_{\breve{\tau}}\right\|_{E} \leq\left\|u-u_{\hat{\tau}}\right\|_{E}$, and

$$
\# \breve{\tau}-\# \tau \leq \# \hat{\tau}-\# \tau_{0} .
$$

To see the last statement, note that each simplex in $\breve{\tau}$ that is not in $\tau$ is in $\hat{\tau}$. Therefore, since $\tau \supset \tau_{0}$, the number of bisections needed to create $\breve{\tau}$ from $\tau$, whose number is equal to $\# \breve{\tau}-\# \tau$, is not larger than the number of bisections needed to create $\hat{\tau}$ from $\tau_{0}$, whose number is equal to \# $\hat{\tau}-\# \tau_{0}$.

With $G=G(\tau, \breve{\tau})$ from Proposition 4.2 , we have

$$
\begin{aligned}
C_{1}^{2} \sum_{T \in G} \eta_{T}\left(f^{1}, f^{2}, u_{\tau}\right) & \geq\left\|u_{\breve{\tau}}-u_{\tau}\right\|_{E}^{2}=\left\|u-u_{\tau}\right\|_{E}^{2}-\left\|u-u_{\breve{\tau}}\right\|_{E}^{2} \\
& \geq \frac{C_{1}^{2} \theta^{2}}{c_{2}^{2}}\left\|u-u_{\tau}\right\|_{E}^{2} \geq C_{1}^{2} \theta^{2} \mathcal{E}\left(\tau, f^{1}, \boldsymbol{f}^{2}, u_{\tau}\right)^{2}
\end{aligned}
$$

by (4.6). By construction of $F$, we conclude that

$$
\# F \lesssim \# G \lesssim \# \breve{\tau}-\# \tau \leq \# \hat{\tau}-\# \tau_{0},
$$

which completes the proof. 
The idea of the goal-oriented AFEM will be to mark sets of simplices for refinement corresponding to both primal and dual problems, and then to perform the actual refinement corresponding to that set of marked simplices that has the smallest cardinality. In order to assess the quality of the method, we first introduce the approximation classes $\mathcal{A}^{s}$.

For $s>0$, we define

$$
\mathcal{A}^{s}=\left\{u \in H_{0}^{1}(\Omega):|u|_{\mathcal{A}^{s}}:=\sup _{\varepsilon>0} \varepsilon \inf _{\left\{\tau:\left\|u-u_{\tau}\right\|_{E} \leq \varepsilon\right\}}\left[\# \tau-\# \tau_{0}\right]^{s}<\infty\right\}
$$

and equip it with norm $\|u\|_{\mathcal{A}^{s}}:=\|u\|_{E}+|u|_{\mathcal{A}^{s}}$. So $\mathcal{A}^{s}$ is the class of functions that can be approximated within any given tolerance $\varepsilon>0$ in \|\|$_{E}$ by a continuous piecewise polynomial of degree $p$ on a partition $\tau$ with $\# \tau-\# \tau_{0} \leq \varepsilon^{-1 / s}|u|_{\mathcal{A}^{s}}^{1 / s}$.

Remark 5.4. Although in the definition of $\mathcal{A}^{s}$ we consider only conforming descendants $\tau$ of $\tau_{0}$, in view of Remark 3.2, we note that these approximation classes would remain the same if we would replace $\tau$ by any descendant $\varrho$ of $\tau_{0}$, conforming or not.

While the $\mathcal{A}^{s}$ contain $\mathbb{V}_{\tau}$ for any $s$, and thus are never empty, only the range $s \leq p / n$ is of interest, as even $C^{\infty}$ functions are only guaranteed to belong to $\mathcal{A}^{s}$ for this range. Classical estimates show that for $s \leq p / n, H^{1+p}(\Omega) \cap H_{0}^{1}(\Omega) \subset \mathcal{A}^{s}$, where it is sufficient to consider uniform refinements. The class $\mathcal{A}^{s}$ is much larger than $H^{1+p}(\Omega) \cap H_{0}^{1}(\Omega)$, which is the reason to consider adaptive methods in the first place. A (near) characterization of $\mathcal{A}^{s}$ for $s \leq p / n$ in terms of Besov spaces can be found in [BDDP02] (although there the case $n=2$ and $p=1$ is considered, results easily generalize).

We now consider the following adaptive algorithm:

$\operatorname{GOAFEM}\left[f^{1}, \boldsymbol{f}^{2}, g^{1}, \boldsymbol{g}^{2}, \varepsilon\right] \rightarrow\left[\tau_{n}, u_{\tau_{n}}, z_{\tau_{n}}\right]$

$\%$ For this preliminary version of the goal-oriented AFEM,

$\%$ it is assumed that $f^{1}, g^{1} \in \mathbb{V}_{\tau_{0}}^{*}$ and $\boldsymbol{f}^{2}, \boldsymbol{g}^{2} \in\left[\mathbb{V}_{\tau_{0}}^{*}\right]^{n}$.

$k:=0$

while $C_{1} \mathcal{E}\left(\tau_{k}, f^{1}, \boldsymbol{f}^{2}, u_{\tau_{k}}\right) \cdot C_{1} \mathcal{E}\left(\tau_{k}, g^{1}, \boldsymbol{g}^{2}, z_{\tau_{k}}\right)>\varepsilon$ do

$F_{p}:=\operatorname{MARK}\left[\tau_{k}, f^{1}, \boldsymbol{f}^{2}, u_{\tau_{k}}\right]$

$F_{d}:=\operatorname{MARK}\left[\tau_{k}, g^{1}, \boldsymbol{g}^{2}, z_{\tau_{k}}\right]$

With $F$ being the smallest of $F_{p}$ and $F_{d}, \tau_{k+1}:=\operatorname{REFINE}\left[\tau_{k}, F\right]$

$k:=k+1$

end do

$n:=k$

TheOREM 5.5. Let $f^{1}, g^{1} \in \mathbb{V}_{\tau_{0}}^{*}$ and $\boldsymbol{f}^{2}, \boldsymbol{g}^{2} \in\left[\mathbb{V}_{\tau_{0}}^{*}\right]^{n}$. Then $\left[\tau_{n}, u_{\tau_{n}}, z_{\tau_{n}}\right]=$ $\operatorname{GOAFEM}\left[f^{1}, \boldsymbol{f}^{2}, g^{1}, \boldsymbol{g}^{2}, \varepsilon\right]$ terminates, and $\left\|u-u_{\tau_{n}}\right\|_{E}\left\|z-z_{\tau_{n}}\right\|_{E} \leq \varepsilon$. If $u \in \mathcal{A}^{s}$ and $z \in \mathcal{A}^{t}$, then

$$
\# \tau_{n}-\# \tau_{0} \lesssim \varepsilon^{-1 /(s+t)}\left(|u|_{\mathcal{A}^{s}}|z|_{\mathcal{A}^{t}}\right)^{1 /(s+t)}
$$

dependent only on $\tau_{0}$, and on $s$ or $t$ when they tend to 0 or $\infty$.

Remark 5.6. Assuming only that $u \in \mathcal{A}^{s}$ and $z \in \mathcal{A}^{t}$, given a partition $\tau$, the generally smallest upper bound for the product of the errors in energy norm in primal and dual solutions that can be expected is $\left[\# \tau-\# \tau_{0}\right]^{-s}|u|_{\mathcal{A}^{s}}\left[\# \tau-\# \tau_{0}\right]^{-t}|z|_{\mathcal{A}^{t}}$. Setting this expression equal to $\varepsilon$, one finds $\# \tau-\# \tau_{0}=\varepsilon^{-1 /(s+t)}\left(|u|_{\mathcal{A}^{s}}|z|_{\mathcal{A}^{t}}\right)^{1 /(s+t)}$. 
We conclude that the partition produced by GOAFEM is at most a constant factor larger than the generally smallest partition $\tau$ for which $\left\|u-u_{\tau}\right\|_{E}\left\|z-z_{\tau}\right\|_{E}$ is less than the prescribed tolerance.

Proof. Let $E_{k}:=\left\|u-u_{\tau_{k}}\right\|_{E}\left\|z-z_{\tau_{k}}\right\|_{E}$. Then $E_{k+1} \leq\left[1-\frac{c_{2}^{2} \theta^{2}}{C_{1}^{2}}\right]^{\frac{1}{2}} E_{k}$ by (5.4), and $c_{2} \mathcal{E}\left(\tau_{k}, f^{1}, \boldsymbol{f}^{2}, u_{\tau_{k}}\right) c_{2} \mathcal{E}\left(\tau_{k}, g^{1}, \boldsymbol{g}^{2}, z_{\tau_{k}}\right) \leq E_{k}$ by (4.6). So GOAFEM $\left[f^{1}, \boldsymbol{f}^{2}, g^{1}, \boldsymbol{g}^{2}, \varepsilon\right]$ terminates, with $E_{n} \leq C_{1} \mathcal{E}\left(\tau_{n}, f^{1}, \boldsymbol{f}^{2}, u_{\tau_{n}}\right) C_{1} \mathcal{E}\left(\tau_{n}, g^{1}, \boldsymbol{g}^{2}, z_{\tau_{n}}\right) \leq \varepsilon$ by (4.4).

With $F_{k}$ being the set of marked cells inside the $k$ th call of REFINE, Lemma 5.3 and the assumptions $u \in \mathcal{A}^{s}, z \in \mathcal{A}^{t}$ show that

$$
\begin{aligned}
\# F_{k} & \leq \min \left\{\left[1-\frac{C_{1}^{2} \theta^{2}}{c_{2}^{2}}\right]^{-\frac{1}{2 s}}\left\|u-u_{\tau_{k-1}}\right\|_{E}^{-1 / s}|u|_{\mathcal{A}^{s}}^{1 / s},\left[1-\frac{C_{1}^{2} \theta^{2}}{c_{2}^{2}}\right]^{-\frac{1}{2 t}}\left\|z-z_{\tau_{k-1}}\right\|_{E}^{-1 / t}|z|_{\mathcal{A}^{t}}^{1 / t}\right\} \\
& \lesssim \min \left\{\left\|u-u_{\tau_{k-1}}\right\|_{E}^{-1 / s}|u|_{\mathcal{A}^{s}}^{1 / s},\left\|z-z_{\tau_{k-1}}\right\|_{E}^{-1 / t}|z|_{\mathcal{A}^{t}}^{1 / t}\right\} \\
& \leq \max _{\delta \eta \geq E_{k-1}} \min \left\{\delta^{-1 / s}|u|_{\mathcal{A}^{s}}^{1 / s}, \eta^{-1 / t}|z|_{\mathcal{A}^{t}}^{1 / t}\right\}=E_{k-1}^{-1 /(s+t)}\left(|u|_{\mathcal{A}^{s}}|z|_{\mathcal{A}^{t}}\right)^{1 /(s+t)} .
\end{aligned}
$$

The partition $\tau_{k}$ is the smallest conforming refinement of the generally nonconforming $\varrho_{k}$, defined as the smallest refinement of $\tau_{k-1}$ which is a full refinement with respect to all $T \in F_{k}$. From Theorem 3.1, \# $\varrho_{k}-\# \tau_{k-1} \lesssim \# F_{k}$, the majorized linear convergence of $k \mapsto E_{k-1}$, and $E_{n-1}>\frac{c_{2}^{2}}{C_{1}^{2}} \varepsilon$, we conclude that

$$
\begin{aligned}
\# \tau_{n}-\# \tau_{0} & \lesssim \sum_{k=1}^{n} \# F_{k} \lesssim E_{n-1}^{-1 /(s+t)}\left(|u|_{\mathcal{A}^{s}}|z|_{\mathcal{A}^{t}}\right)^{1 /(s+t)} \\
& \lesssim \varepsilon^{-1 /(s+t)}\left(|u|_{\mathcal{A}^{s}}|z|_{\mathcal{A}^{t}}\right)^{1 /(s+t)} .
\end{aligned}
$$

6. A practical goal-oriented AFEM. So far, we assumed that $f=f^{1}+$ $\operatorname{div} \boldsymbol{f}^{2}, g=g^{1}+\operatorname{div} \boldsymbol{g}^{2}$, with $f^{1}, g^{1} \in \mathbb{V}_{\tau}^{*}, \boldsymbol{f}^{2}, \boldsymbol{g}^{2} \in\left[\mathbb{V}_{\tau}^{*}\right]^{n}$ for any partition $\tau$ that we encountered; i.e., we assumed that $f^{1}, g^{1} \in \mathbb{V}_{\tau_{0}}^{*}, \boldsymbol{f}^{2}, \boldsymbol{g}^{2} \in\left[\mathbb{V}_{\tau_{0}}^{*}\right]^{n}$. From now on, given a partition $\tau$, we will approximate $f, g \in H^{-1}(\Omega)$ by $f_{\tau^{\prime}}^{1}+\operatorname{div} \boldsymbol{f}_{\tau^{\prime}}^{2}, g_{\tau^{\prime}}^{1}+\operatorname{div} \boldsymbol{g}_{\tau^{\prime}}^{2}$, respectively, where $f_{\tau^{\prime}}^{1}, g_{\tau^{\prime}}^{1} \in \mathbb{V}_{\tau^{\prime}}^{*}, \boldsymbol{f}_{\tau^{\prime}}^{2}, \boldsymbol{g}_{\tau^{\prime}}^{2} \in\left[\mathbb{V}_{\tau^{\prime}}^{*}\right]^{n}$ and either $\tau^{\prime}=\tau$ or, when it is needed to have a smaller approximation error, $\tau^{\prime} \supset \tau$. We will set

$$
f_{\tau^{\prime}}:=f_{\tau^{\prime}}^{1}+\operatorname{div} \boldsymbol{f}_{\tau^{\prime}}^{2}, \quad g_{\tau^{\prime}}:=g_{\tau^{\prime}}^{1}+\operatorname{div} \boldsymbol{g}_{\tau^{\prime}}^{2} .
$$

To be able to distinguish between primal or dual solutions corresponding to different right-hand sides, we introduce operators $L: H_{0}^{1}(\Omega) \rightarrow H^{-1}(\Omega)$ by $(L v)(w)=$ $a(v, w)\left(v, w \in H_{0}^{1}(\Omega)\right)$, and $L_{\tau}: \mathbb{V}_{\tau} \rightarrow \mathbb{V}_{\tau}^{\prime}$ by $\left(L_{\tau} v_{\tau}\right)\left(w_{\tau}\right)=a\left(v_{\tau}, w_{\tau}\right)\left(v_{\tau}, w_{\tau} \in \mathbb{V}_{\tau}\right)$. The solutions $u, z, u_{\tau}, z_{\tau}$ of (2.1), (2.2), (4.1), (4.2) can now be written as $L^{-1} f$, $\left(L^{\prime}\right)^{-1} g, L_{\tau}^{-1} f,\left(L_{\tau}^{\prime}\right)^{-1} g$, respectively. Since in our case $L^{\prime}=L$ and $L_{\tau}^{\prime}=L_{\tau}$, for notational convenience we will drop the prime. Note that $\|L \cdot\|_{E^{\prime}}=\|\cdot\|_{E},\left\|L_{\tau}^{-1}\right\|_{E^{\prime} \rightarrow E} \leq 1$, and $\left\|\left(L^{-1}-L_{\tau}^{-1}\right)\right\|_{E^{\prime} \rightarrow E} \leq 1$.

Furthermore, in view of controlling the cost of our adaptive solver, from now on we will solve the arising Galerkin systems only approximately.

The following lemma generalizes upon Lemma 5.3, relaxing both the condition that the right-hand side is in $\mathbb{V}_{\tau}^{*}+\operatorname{div}\left[\mathbb{V}_{\tau}^{*}\right]^{n}$ and the assumption that we have the exact Galerkin solution available, assuming that the deviations from that ideal situation are sufficiently small in a relative sense.

Lemma 6.1 (see [Ste07, Lemmas 6.1 and 6.2]). There exist positive constants $\omega=\omega\left(\theta, C_{1}, c_{2}\right)$ and $\lambda=\lambda\left(\omega, C_{1}, c_{2}\right)$ such that for any $f \in H^{-1}(\Omega)$, partition $\tau$, 
$f_{\tau}^{1} \in \mathbb{V}_{\tau}^{*}, \boldsymbol{f}_{\tau}^{2} \in\left[\mathbb{V}_{\tau}^{*}\right]^{n}, \bar{u}_{\tau} \in \mathbb{V}_{\tau}$ with

$$
\left\|f-f_{\tau}\right\|_{E^{\prime}}+\left\|L_{\tau}^{-1} f_{\tau}-\bar{u}_{\tau}\right\|_{E} \leq \omega \mathcal{E}\left(\tau, f_{\tau}^{1}, \boldsymbol{f}_{\tau}^{2}, \bar{u}_{\tau}\right),
$$

$F:=\operatorname{MARK}\left[\tau, f_{\tau}^{1}, f_{\tau}^{2}, \bar{u}_{\tau}\right]$ satisfies

$$
\# F \lesssim \# \hat{\tau}-\# \tau_{0}
$$

for any partition $\hat{\tau}$ for which

$$
\left\|u-u_{\hat{\tau}}\right\|_{E} \leq \lambda\left\|u-\bar{u}_{\tau}\right\|_{E} .
$$

Furthermore, given a

$$
\mu \in\left(\left[1-\frac{c_{2}^{2} \theta^{2}}{C_{1}^{2}}\right]^{\frac{1}{2}}, 1\right)
$$

there exists an $\omega=\omega\left(\mu, \theta, C_{1}, c_{2}\right)>0$, such that if (6.1) is valid for this $\omega$, and for $\tau^{\prime} \supseteq \operatorname{REFINE}[\tau, F], f_{\tau^{\prime}} \in H^{-1}(\Omega)$ and $\bar{u}_{\tau^{\prime}} \in \mathbb{V}_{\tau^{\prime}}$,

$$
\left\|f-f_{\tau^{\prime}}\right\|_{E^{\prime}}+\left\|L_{\tau^{\prime}}^{-1} f_{\tau^{\prime}}-\bar{u}_{\tau^{\prime}}\right\|_{E} \leq \omega \mathcal{E}\left(\tau, f_{\tau}^{1}, \boldsymbol{f}_{\tau}^{2}, \bar{u}_{\tau}\right),
$$

then

$$
\left\|u-\bar{u}_{\tau^{\prime}}\right\|_{E} \leq \mu\left\|u-\bar{u}_{\tau}\right\|_{E} .
$$

For solving the Galerkin systems approximately, we assume that we have an iterative solver of optimal type available:

$\operatorname{GALSOLVE}\left[\tau, f_{\tau}, u_{\tau}^{(0)}, \delta\right] \rightarrow \bar{u}_{\tau}$ $\% f_{\tau} \in\left(\mathbb{V}_{\tau}\right)^{\prime}$ and $u_{\tau}^{(0)} \in \mathbb{V}_{\tau}$, the latter being an initial approximation for an $\%$ iterative solver. The output $\bar{u}_{\tau} \in \mathbb{V}_{\tau}$ satisfies

$$
\left\|L_{\tau}^{-1} f_{\tau}-\bar{u}_{\tau}\right\|_{E} \leq \delta .
$$

$\%$ The call requires $\lesssim \max \left\{1, \log \left(\delta^{-1}\left\|L_{\tau}^{-1} f_{\tau}-u_{\tau}^{(0)}\right\|_{E}\right)\right\} \# \tau$

$\%$ arithmetic operations.

Multigrid methods with local smoothing, or their additive variants (Bramble-Pasciak$\mathrm{Xu}$ ) as preconditioners in conjugate gradients, are known to be of this type.

A routine called $\mathbf{R H S} \mathbf{S}_{f}$, and analogously $\mathbf{R H S}_{g}$, will be needed to find a sufficiently accurate approximation to the right-hand side $f$ of the form $f_{\tau}^{1}+\operatorname{div} \boldsymbol{f}_{\tau}^{2}$ with $f_{\tau}^{1} \in \mathbb{V}_{\tau}^{*}, \boldsymbol{f}_{\tau}^{2} \in\left[\mathbb{V}_{\tau}^{*}\right]^{n}$. Since this might not be possible with respect to the current partition, a call of $\mathbf{R H S}_{f}$ may result in further refinement.

$\mathbf{R H S}_{f}[\tau, \delta] \rightarrow\left[\tau^{\prime}, f_{\tau^{\prime}}^{1}, \boldsymbol{f}_{\tau^{\prime}}^{2}\right]$

$\% \delta>0$. The output consists of $f_{\tau^{\prime}}^{1} \in \mathbb{V}_{\tau^{\prime}}^{*}$ and $\boldsymbol{f}_{\tau^{\prime}}^{2} \in\left[\mathbb{V}_{\tau^{\prime}}^{*}\right]^{n}$, where $\tau^{\prime}=\tau$ or, $\%$ if necessary, $\tau^{\prime} \supset \tau$, such that $\left\|f-f_{\tau^{\prime}}\right\|_{E^{\prime}} \leq \delta$.

Assuming that $u \in \mathcal{A}^{s}$ for some $s>0$, the cost of approximating the righthand side $f$ using $\mathbf{R H S}_{f}$ will generally not dominate the other costs of our adaptive 
method only if there is some constant $c_{f}$ such that for any $\delta>0$ and any partition $\tau$, for $\left[\tau^{\prime}, \cdot, \cdot\right]:=\mathbf{R H S}_{f}[\tau, \delta]$, it holds that

$$
\# \tau^{\prime}-\# \tau \leq c_{f}^{1 / s} \delta^{-1 / s},
$$

and the number of arithmetic operations required by the call is $\lesssim \# \tau^{\prime}$. We will refer to such an $\mathbf{R H S}_{f}$ as $s$-optimal with constant $c_{f}$. Obviously, given $s$, such a routine can exist only when $f \in \overline{\mathcal{A}}^{s}$, defined by

$$
\overline{\mathcal{A}}^{s}=\left\{f \in H^{-1}(\Omega): \sup _{\varepsilon>0} \varepsilon \inf _{\left\{\tau: \inf _{f_{\tau}^{1} \in \mathbb{V}_{\tau}^{*}, f_{\tau}^{2} \in\left[\mathbb{V}_{\tau}^{*}\right]^{n}}\left\|f-f_{\tau}\right\|_{E^{\prime}} \leq \varepsilon\right\}}\left[\# \tau-\# \tau_{0}\right]^{s}<\infty\right\} .
$$

On the one hand, $u \in \mathcal{A}^{s}$ implies that $f \in \overline{\mathcal{A}}^{s}$. Indeed, for any partition $\tau$, let $\boldsymbol{f}_{\tau}^{2}:=-\boldsymbol{A} \nabla u_{\tau}$. Then $\boldsymbol{f}_{\tau}^{2} \in\left[\mathbb{V}_{\tau}^{*}\right]^{n}$ and $\left\|f-\operatorname{div} \boldsymbol{f}_{\tau}^{2}\right\|_{E^{\prime}}=\left\|u-u_{\tau}\right\|_{E}$. On the other hand, knowing that $f \in \overline{\mathcal{A}}^{s}$ is a different thing than knowing how to construct suitable approximations. If $s \in\left[\frac{1}{n}, \frac{p+1}{n}\right]$ and $f \in H^{s n-1}(\Omega)$, then the best approximations $f_{\tau}^{1}$ to $f$ from $\mathbb{V}_{\tau}^{*}$ with respect to $L_{2}(\Omega)$ using uniform refinements $\tau$ of $\tau_{0}$ are known to converge with the required rate. For general $f \in \overline{\mathcal{A}}^{s}$, however, a realization of a suitable routine $\mathbf{R H S}_{f}$ has to depend on the functional $f$ at hand.

Remark 6.2. When $u$ and $f$ are smooth, then $u \in \mathcal{A}^{p / n}$ and $f \in \overline{\mathcal{A}}^{(p+1) / n}$. Indeed, $u$ is approximated by piecewise polynomials of degree $p$, and $f$ by those of degree $p-1$ (apart from possible approximations from $\operatorname{div}\left[\mathbb{V}_{\tau}^{*}\right]^{n}$ ), whereas the errors are measured in $H_{0}^{1}(\Omega)$ or $H^{-1}(\Omega)$, respectively. Also for less smooth $u$ and $f$, one can expect that usually $u \in \mathcal{A}^{s}$ and $f \in \overline{\mathcal{A}}^{s^{\prime}}$ for some $s^{\prime}>s$.

In our adaptive method, given some partition $\tau$, for both computing the error estimator and setting up the Galerkin system, we will replace $f$ by an approximation from $\mathbb{V}_{\tau^{\prime}}^{*}+\operatorname{div}\left[\mathbb{V}_{\tau^{\prime}}^{*}\right]^{n}$ where $\tau^{\prime} \supseteq \tau$ (and similarly for $g$ ). This has the advantages that we can consider $f \notin L_{2}(\Omega)+\operatorname{div}_{\mathbb{W}_{\tau}^{*}}^{*}$, for which thus the error estimator is not defined, and that we don't have to worry about quadrature errors in various places in the algorithm.

Assuming $f \in L_{2}(\Omega)+\operatorname{div} \mathbb{W}_{\tau}^{n}$ for any $\tau$, another option, followed in [MNS00], is not to replace $f$ by an approximation, but to check whether, on the current partition, the error in the best approximation for $f$ from $\mathbb{V}_{\tau}^{*}\left(+\operatorname{div}\left[\mathbb{V}_{\tau}^{*}\right]^{n}\right)$, called data oscillation, is sufficiently small relative to the error in the current approximation to $u$, and, if not, to refine $\tau$ to achieve this. Convergence of this approach was shown, and it can be expected that by applying suitable quadrature and inexact Galerkin solves, optimal computational complexity can be shown as well. The observations at the beginning of this remark indicate that "usually," at least asymptotically, there will be no refinements needed to reduce the data oscillation. This explains why common adaptive methods that ignore data oscillation usually converge with optimal rates.

In addition to being $s$-optimal, we will have to assume that $\mathbf{R H S}_{f}$ is linearly convergent, by which we mean that for any $d \in(0,1)$, there exists a $D>0$ such that for any $\delta>0$, partitions $\tau$ and $\tau^{\prime} \supseteq \hat{\tau}$ where $[\hat{\tau}, \cdot, \cdot]:=\mathbf{R H S}_{f}[\tau, \delta]$, the output $\left[\tau^{\prime \prime}, \cdot, \cdot\right]:=\mathbf{R H S}_{f}\left[\tau^{\prime}, d \delta\right]$ satisfies $\# \tau^{\prime \prime} \leq D \# \tau^{\prime}$.

Remark 6.3. Usually, a realization of $[\hat{\tau}, \cdot, \cdot]:=\mathbf{R H S}_{f}[\tau, \delta]$ will be based on the selection of $\hat{\tau}$ such that an upper bound for the error is less than the prescribed tolerance. Since this upper bound will be an algebraically decreasing function of $\# \hat{\tau}-\# \tau_{0}$, linear convergence is obtained.

We now have the ingredients in hand to define our practical adaptive goal-oriented finite element routine GOAFEM. Compared to the idealized version from the previous section, we will have to deal with the fact that when solving the Galerkin systems 
only inexactly, and applying inexact right-hand sides, $C_{1}$ times the a posteriori error estimator $\mathcal{E}(\cdot)$ is not necessarily an upper bound for the energy norm of the error. We have to add correction terms to obtain an upper bound. Furthermore, after applying REFINE on either the primal or dual side, we have to specify a tolerance for the error in the new approximation of the right-hand side and in that of the new approximate Galerkin solution. In order to know that a subsequent REFINE results in an error reduction, in view of Lemma 6.1 we would like to choose this tolerance smaller than $\omega$ times the new error estimator, which, however, is not known yet. Although we can expect that usually the new estimator is only some moderate factor less than the existing one, it cannot be excluded that the new estimator is arbitrarily small, e.g., when we happen to have reached a partition on which the solution can be exactly represented. In this case, an error reduction is immediate, and so we don't have to rely on REFINE to achieve it.

$\operatorname{GOAFEM}\left[f, g, \delta_{p}, \delta_{d}, \varepsilon\right] \rightarrow\left[\tau, \bar{u}_{\tau}, \bar{z}_{\tau}\right]$

$\%$ Let $\omega \in\left(0, c_{2}\right)$ be a constant not larger than the constants $\omega\left(\theta, C_{1}, c_{2}\right)$ and $\% \omega\left(\mu, \theta, C_{1}, c_{2}\right)$ for some $\mu \in\left(\left[1-\frac{c^{2} \theta^{2}}{C_{1}^{2}}\right]^{\frac{1}{2}}, 1\right)$ mentioned in Lemma 6.1 .

$\%$ Let $0<\beta<\left[\left(\frac{2+3 C_{1} c_{2}^{-1}}{2+C_{1} c_{2}^{-1}}+C_{1} c_{2}^{-1}\right)\left(2+C_{1}\left(c_{2}^{-1}+2 \omega^{-1}\right)\right)\right]^{-1}$ be a constant. $\tau:=\tau_{0},\left[\tau_{p}, f_{\tau_{p}}^{1}, \boldsymbol{f}_{\tau_{p}}^{2}\right]:=\mathbf{R H S}_{f}\left[\tau, \delta_{p}\right],\left[\tau_{d}, g_{\tau_{d}}^{1}, \boldsymbol{g}_{\tau_{d}}^{2}\right]:=\mathbf{R H S}_{g}\left[\tau, \delta_{d}\right]$ $\bar{u}_{\tau_{p}}:=\bar{z}_{\tau_{d}}:=0$

do

$$
\begin{aligned}
& \bar{u}_{\tau_{p}}:=\operatorname{GALSOLVE}\left[\tau_{p}, f_{\tau_{p}}, \bar{u}_{\tau_{p}}, \delta_{p}\right] \\
& \bar{z}_{\tau_{d}}:=\text { GALSOLVE }\left[\tau_{d}, g_{\tau_{d}}, \bar{z}_{\tau_{d}}, \delta_{d}\right] \\
& \sigma_{p}:=\left(2+C_{1} c_{2}^{-1}\right) \delta_{p}+C_{1} \mathcal{E}\left(\tau_{p}, f_{\tau_{p}}^{1}, \boldsymbol{f}_{\tau_{p}}^{2}, \bar{u}_{\tau_{p}}\right) \\
& \sigma_{d}:=\left(2+C_{1} c_{2}^{-1}\right) \delta_{d}+C_{1} \mathcal{E}\left(\tau_{d}, g_{\tau_{p}}^{1}, \boldsymbol{g}_{\tau_{p}}^{2}, \bar{z}_{\tau_{d}}\right) \\
& \text { if } \sigma_{p} \sigma_{d} \leq \varepsilon \text { then } \tau:=\tau_{p} \cup \tau_{d}, \bar{u}_{\tau}:=\bar{u}_{\tau_{p}}, \bar{z}_{\tau}:=\bar{z}_{\tau_{d}} \text { stop endif } \\
& \text { if } 2 \delta_{p} \leq \omega \mathcal{E}\left(\tau_{p}, f_{\tau_{p}}^{1}, \boldsymbol{f}_{\tau_{p}}^{2}, \bar{u}_{\tau_{p}}\right) \text { then } F_{p}:=\operatorname{MARK}\left[\tau, f_{\tau_{p}}^{1}, \boldsymbol{f}_{\tau_{p}}^{2}, \bar{u}_{\tau_{p}}\right] \\
& \text { else } F_{p}:=\emptyset \text { endif } \\
& \text { if } 2 \delta_{d} \leq \omega \mathcal{E}\left(\tau_{d}, g_{\tau_{p}}^{1}, \boldsymbol{g}_{\tau_{p}}^{2}, \bar{z}_{\tau_{d}}\right) \text { then } F_{d}:=\operatorname{MARK}\left[\tau, g_{\tau_{p}}^{1}, \boldsymbol{g}_{\tau_{p}}^{2}, \bar{z}_{\tau_{d}}\right] \\
& \text { else } F_{d}:=\emptyset \text { endif } \\
& \text { if } \# \tau_{p}-\# \tau+\# F_{p} \leq \# \tau_{d}-\# \tau+\# F_{d} \\
& \text { then } \tau:=\operatorname{REFINE}\left[\tau_{p}, F_{p}\right], \delta_{p}:=\min \left(\delta_{p}, \beta \sigma_{p}\right) \\
& {\left[\tau_{p}, f_{\tau_{p}}^{1}, \boldsymbol{f}_{\tau_{p}}^{2}\right]:=\mathbf{R H S}_{f}\left[\tau, \delta_{p}\right], \tau_{d}:=\tau \cup \tau_{d}} \\
& \text { else } \tau:=\mathbf{R E F I N E}\left[\tau_{d}, F_{d}\right], \delta_{d}:=\min \left(\delta_{d}, \beta \sigma_{d}\right) \\
& \tau_{p}:=\tau \cup \tau_{p},\left[\tau_{d}, g_{\tau_{p}}^{1}, \boldsymbol{g}_{\tau_{p}}^{2}\right]:=\mathbf{R H S}_{g}\left[\tau, \delta_{d}\right] \\
& \text { endif } \\
& \text { enddo }
\end{aligned}
$$

TheOREM 6.4. $\left[\tau, \bar{u}_{\tau}, \bar{z}_{\tau}\right]=\operatorname{GOAFEM}\left[f, g, \underline{\delta}_{p}, \underline{\delta}_{d}, \varepsilon\right]$ terminates, and

$$
\left\|u-\bar{u}_{\tau}\right\|_{E}\left\|z-\bar{z}_{\tau}\right\|_{E} \leq \varepsilon .
$$

If $u \in \mathcal{A}^{s}, z \in \mathcal{A}^{t}, \mathbf{R H S}_{f}$ (RHS $\mathbf{R H}_{g}$ ) is s-optimal (t-optimal) with constant $c_{f}\left(c_{g}\right)$, $\underline{\delta}_{p}>c_{f}$, and $\underline{\delta}_{d}>c_{g}$, then

$$
\# \tau \lesssim \# \tau_{0}+\varepsilon^{-1 /(s+t)}\left[\left(|u|_{\mathcal{A}^{s}}^{1 / s}+c_{f}^{1 / s}\right)^{s}\left(|z|_{\mathcal{A}^{t}}^{1 / t}+c_{g}^{1 / t}\right)^{t}\right]^{1 /(s+t)} .
$$

If, additionally, $\|f\|_{E^{\prime}} \lesssim \underline{\delta}_{p},\|g\|_{E^{\prime}} \lesssim \underline{\delta}_{d}$, and $\underline{\delta}_{p} \underline{\delta}_{d} \lesssim\left\|u-u_{\tau_{0}}\right\|_{E}\left\|z-z_{\tau_{0}}\right\|_{E}+\varepsilon$, then the number of arithmetic operations and storage locations required by the call 
are bounded by some absolute multiple of the same expression. The constant factors involved in these bounds may depend only on $\tau_{0}$, and on $s$ or $t$ when they tend to 0 or $\infty$, and concerning the cost, on the constants involved in the additional assumptions.

Remark 6.5. The condition $\underline{\delta}_{p}>c_{f}$ implies that for a call $\left[\tau^{\prime}, \cdot, \cdot\right]=\mathbf{R H S}\left[\tau, \underline{\delta}_{p}\right]$, we have $\tau^{\prime}=\tau$.

Proof. We start with collecting a few useful estimates. At evaluation of $\sigma_{p}$, by (4.4) and Proposition 4.6, we have

$$
\begin{aligned}
\left\|u-\bar{u}_{\tau_{p}}\right\|_{E} & \leq\left\|u-L^{-1} f_{\tau_{p}}\right\|_{E}+\left\|\left(L^{-1}-L_{\tau_{p}}^{-1}\right) f_{\tau_{p}}\right\|_{E}+\left\|L_{\tau_{p}}^{-1} f_{\tau_{p}}-\bar{u}_{\tau_{p}}\right\|_{E} \\
& \leq \delta_{p}+C_{1} \mathcal{E}\left(\tau_{p}, f_{\tau_{p}}^{1}, \boldsymbol{f}_{\tau_{p}}^{2}, L_{\tau_{p}}^{-1} f_{\tau_{p}}\right)+\left\|L_{\tau_{p}}^{-1} f_{\tau_{p}}-\bar{u}_{\tau_{p}}\right\|_{E} \\
& \leq \delta_{p}+C_{1} \mathcal{E}\left(\tau_{p}, f_{\tau_{p}}^{1}, \boldsymbol{f}_{\tau_{p}}^{2}, \bar{u}_{\tau_{p}}\right)+\left(C_{1} c_{2}^{-1}+1\right)\left\|L_{\tau_{p}}^{-1} f_{\tau_{p}}-\bar{u}_{\tau_{p}}\right\|_{E} \\
& \leq\left(2+C_{1} c_{2}^{-1}\right) \delta_{p}+C_{1} \mathcal{E}\left(\tau_{p}, f_{\tau_{p}}^{1}, \boldsymbol{f}_{\tau_{p}}^{2}, \bar{u}_{\tau_{p}}\right)=: \sigma_{p}
\end{aligned}
$$

and, by Corollary 4.5,

$$
\begin{aligned}
\mathcal{E}\left(\tau_{p}, f_{\tau_{p}}^{1}, \boldsymbol{f}_{\tau_{p}}^{2}, \bar{u}_{\tau_{p}}\right) & \leq c_{2}^{-1}\left\|L^{-1} f_{\tau_{p}}-\bar{u}_{\tau_{p}}\right\|_{E} \\
& \leq c_{2}^{-1}\left[\left\|u-u_{\tau_{p}}\right\|_{E}+\left\|\left(L^{-1}-L_{\tau_{p}}^{-1}\right)\left(f_{\tau_{p}}-f\right)\right\|_{E}+\left\|L_{\tau_{p}}^{-1} f_{\tau_{p}}-\bar{u}_{\tau_{p}}\right\|_{E}\right] \\
& \leq c_{2}^{-1}\left\|u-u_{\tau_{p}}\right\|_{E}+c_{2}^{-1} 2 \delta_{p} .
\end{aligned}
$$

So if $2 \delta_{p} \leq \omega \mathcal{E}\left(\tau_{p}, f_{\tau_{p}}^{1}, \boldsymbol{f}_{\tau_{p}}^{2}, \bar{u}_{\tau_{p}}\right)$, then $\mathcal{E}\left(\tau_{p}, f_{\tau_{p}}^{1}, \boldsymbol{f}_{\tau_{p}}^{2}, \bar{u}_{\tau_{p}}\right) \leq\left[c_{2}-\omega\right]^{-1}\left\|u-u_{\tau_{p}}\right\|_{E}$, and so

$$
\sigma_{p} \leq D\left\|u-u_{\tau_{p}}\right\|_{E}, \quad \text { where } D:=\frac{\left(1+\frac{1}{2} C_{1} c_{2}^{-1}\right) \omega+C_{1}}{c_{2}-\omega} .
$$

Now we are ready to show majorized linear convergence of $\sigma_{p} \sigma_{d}$. Consider any two instances $\sigma_{p}^{(A)}$ and $\sigma_{p}^{(B)}$ of $\sigma_{p}$, where $\sigma_{p}^{(A)}$ has been computed preceding $\sigma_{p}^{(B)}$. With $\delta_{p}^{(A)}, \delta_{p}^{(B)}$ and $\tau_{p}^{(A)}, \tau_{p}^{(B)}$ being the corresponding tolerances and partitions, from $(6.3), \delta_{p}^{(B)} \leq \delta_{p}^{(A)}$ and $\tau_{p}^{(B)} \supseteq \tau_{p}^{(A)}$, and so $\left\|u-u_{\tau_{p}^{(B)}}\right\|_{E} \leq\left\|u-\bar{u}_{\tau_{p}^{(A)}}\right\|_{E} \leq \sigma_{p}^{(A)}$ by (6.2), and we have

$$
\begin{aligned}
\sigma_{p}^{(B)} & =\left(2+C_{1} c_{2}^{-1}\right) \delta_{p}^{(B)}+C_{1} \mathcal{E}\left(\tau_{p}^{(B)}, f_{\tau_{p}^{(B)}}^{1}, \boldsymbol{f}_{\tau_{p}^{(B)}}^{2}, \bar{u}_{\tau_{p}^{(B)}}\right) \\
& \leq\left(2+3 C_{1} c_{2}^{-1}\right) \delta_{p}^{(A)}+C_{1} c_{2}^{-1} \sigma_{p}^{(A)} \\
& \leq K \sigma_{p}^{(A)}, \quad \text { where } K:=\frac{2+3 C_{1} c_{2}^{-1}}{2+C_{1} c_{2}^{-1}}+C_{1} c_{2}^{-1} .
\end{aligned}
$$

Let us denote by $\tau_{p}^{(i)}, \delta_{p}^{(i)}, f_{\tau_{p}^{(i)}}^{1}, \boldsymbol{f}_{\tau_{p}^{(i)}}^{2}, \bar{u}_{\tau_{p}^{(i)}}, \sigma_{p}^{(i)}$ the instances of $\tau_{p}, \delta_{p}, f_{\tau_{p}}^{1}, \boldsymbol{f}_{\tau_{p}}^{2}$, $\bar{u}_{\tau_{p}}, \sigma_{p}$ at the moment of the $i$ th call of $\operatorname{REFINE}\left[\tau_{p}, F_{p}\right]$. If $2 \delta_{p}^{(i)}>\omega \mathcal{E}\left(\tau_{p}^{(i)}, f_{\tau_{p}^{(i)}}^{1}\right.$, $\left.\boldsymbol{f}_{\tau_{p}^{(i)}}^{2}, \bar{u}_{\tau_{p}^{(i)}}\right)$, then for any $k<i$,

$$
\sigma_{p}^{(i)}<\left(2+C_{1}\left(c_{2}^{-1}+2 \omega^{-1}\right)\right) \delta_{p}^{(i)} \leq\left(2+C_{1}\left(c_{2}^{-1}+2 \omega^{-1}\right)\right) \beta \sigma_{p}^{(k)} .
$$

If, for some $k \in \mathbb{N}_{0}, 2 \delta_{p}^{(j)} \leq \omega \mathcal{E}\left(\tau_{p}^{(j)}, f_{\tau_{p}^{(j)}}^{1}, f_{\tau_{p}^{(j)}}^{2}, \bar{u}_{\tau_{p}^{(j)}}\right)$ for $j=i, \ldots, i-k$, then by (6.4), Lemma 6.1, where we use that $\delta_{p}^{(j)} \leq \delta_{p}^{(j-1)}$, and (6.2),

$$
\sigma_{p}^{(i)} \leq D\left\|u-\bar{u}_{\tau_{p}^{(i)}}\right\|_{E} \leq D \mu^{k}\left\|u-\bar{u}_{\tau_{p}^{(i-k)}}\right\|_{E} \leq D \mu^{k} \sigma_{p}^{(i-k)} .
$$


Since $\left(2+C_{1}\left(c_{2}^{-1}+2 \omega^{-1}\right)\right) \beta<1 / K$ by definition of $\beta$, from (6.5) we conclude that for any $\alpha \in(0,1)$ there exists an $M$ such that $\sigma_{p}^{(i+M)} \leq \alpha \sigma_{p}^{(i)}$. Since all results derived so far are equally valid on the dual side, by taking $\alpha<1 / K$ we infer that by $2 M$ iterations of the loop inside GOAFEM, the product $\sigma_{p} \sigma_{d}$ is reduced by a factor $\alpha K<1$. Indeed, either $\sigma_{p}$ or $\sigma_{p}$ is reduced by a factor $\alpha$, whereas the other cannot increase by a factor larger than $K$.

Next, we bound the cardinality of the output partition. If GOAFEM terminates as a result of the first evaluation of the test $\sigma_{p} \sigma_{d} \leq \varepsilon$, then by the assumptions that $\underline{\delta}_{p}>c_{f}$ and $\underline{\delta}_{d}>c_{g}$, the output partition $\tau_{p} \cup \tau_{d}=\tau_{0}$. In the following, we consider the case that initially $\sigma_{p} \sigma_{d}>\varepsilon$.

At evaluation of the test $\# \tau_{p}-\# \tau+\# F_{p} \leq \# \tau_{d}-\# \tau+\# F_{d}$, we have

$$
\# \tau_{p}-\# \tau \leq\left(\beta K^{-1} \sigma_{p}\right)^{-1 / s} c_{f}^{1 / s} \text {. }
$$

Indeed, the current $\# \tau_{p}-\# \tau$ is not larger than this difference at the moment of the most recent call of $\mathbf{R H S}_{f}\left[\tau, \delta_{p}\right]$. By the assumption of $\mathbf{R H S}_{f}$ being $s$-optimal, the latter difference was zero when at that time $\delta_{p}>c_{f}$. Otherwise, since $\underline{\delta}_{p}>c_{f}$ by assumption, this $\delta_{p}$ was equal to $\beta$ times the minimum of all values attained by $\sigma_{p}$ up to that moment. Using (6.5) and the fact that $\mathbf{R H S}_{f}$ is $s$-optimal with constant $c_{f}$, we end up with (6.6).

If, at evaluation of the test $\# \tau_{p}-\# \tau+\# F_{p} \leq \# \tau_{d}-\# \tau+\# F_{d}, F_{p} \neq \emptyset$, i.e., if in the preceding lines $2 \delta_{p} \leq \omega \mathcal{E}\left(\tau_{p}, f_{\tau_{p}}^{1}, \boldsymbol{f}_{\tau_{p}}^{2}, \bar{u}_{\tau_{p}}\right)$ and $F_{p}:=\operatorname{MARK}\left[\tau, f_{\tau_{p}}^{1}, \boldsymbol{f}_{\tau_{p}}^{2}, \bar{u}_{\tau_{p}}\right]$, an application of Lemma 6.1 and the assumption that $u \in \mathcal{A}^{s}$ show that then

$$
\# F_{p} \lesssim\left\|u-\bar{u}_{\tau_{p}}\right\|_{E}^{-1 / s}|u|_{\mathcal{A}^{s}}^{1 / s} \lesssim \sigma_{p}^{-1 / s}|u|_{\mathcal{A}^{s}}^{1 / s}
$$

by (6.4).

Clearly, results analogous to (6.6) and (6.7) are valid on the dual side. Now with $\sigma_{p, j}, \sigma_{d, j}$ being the instances of $\sigma_{p}, \sigma_{d}$ at the $j$ th evaluation of the test \# $\tau_{p}-\# \tau+$ $\# F_{p} \leq \# \tau_{d}-\# \tau+\# F_{d}$, with $n$ being the last one, an application of Theorem 3.1 shows that for $\tau$ being the output of the call of REFINE following this last test, being thus the last call of REFINE, we have

$$
\begin{aligned}
\# \tau-\# \tau_{0} & \lesssim \sum_{j=1}^{n} \min \left\{\sigma_{p, j}^{-1 / s}\left(|u|_{\mathcal{A}^{s}}^{1 / s}+c_{f}^{1 / s}\right), \sigma_{d, j}^{-1 / t}\left(|z|_{\mathcal{A}^{t}}^{1 / t}+c_{g}^{1 / t}\right)\right\} \\
& \leq \sum_{j=1}^{n}\left(\sigma_{p, j} \sigma_{d, j}\right)^{-1 /(s+t)}\left[\left(|u|_{\mathcal{A}^{s}}^{1 / s}+c_{f}^{1 / s}\right)^{s}\left(|z|_{\mathcal{A}^{t}}^{1 / t}+c_{g}^{1 / t}\right)^{t}\right]^{1 /(s+t)} \\
& \lesssim \varepsilon^{-1 /(s+t)}\left[\left(|u|_{\mathcal{A}^{s}}^{1 / s}+c_{f}^{1 / s}\right)^{s}\left(|z|_{\mathcal{A}^{t}}^{1 / t}+c_{g}^{1 / t}\right)^{t}\right]^{1 /(s+t)}
\end{aligned}
$$

by the majorized linear convergence of $\left(\sigma_{p, j} \sigma_{d, j}\right)_{j}$ and $\sigma_{p, n} \sigma_{d, n}>\varepsilon$.

Suppose that this last call of REFINE took place on the primal side. Then the output partition of GOAFEM is $\tau_{p} \cup \tau_{d}$, where $\left[\tau_{p}, \cdot, \cdot\right]:=\mathbf{R H S}_{f}\left[\tau, \delta_{p}\right]$ and $\tau_{d}:=\tau \cup \tau_{d}$. As we have seen, if $\delta_{p} \leq c_{f}$, i.e., if possibly $\tau_{p} \supsetneq \tau$, then $\delta_{p}$ is larger than $\beta K^{-1}$ times the current $\sigma_{p}$, which, by its definition, is larger than $2+C_{1} c_{2}^{-1}$ times the previous value of $\delta_{p}$, denoted as $\delta_{p}^{\text {(prev) }}$. A call of $\mathbf{R H S}_{f}\left[\cdot, \delta_{p}^{(\text {prev })}\right]$ has been made inside GOAFEM, and so $\tau \supseteq \tau^{\prime}$ with $\left[\tau^{\prime}, \cdot, \cdot\right]:=\mathbf{R H S}_{f}\left[\cdot, \delta_{p}^{(\text {prev) }}\right]$. The assumption of $\mathbf{R H S}_{f}$ being linearly convergent shows that $\# \tau_{p} \lesssim \# \tau$. 
The current $\# \tau_{d}-\# \tau$ is not larger than this difference at the moment of the last call of $\mathbf{R H S}_{g}$, and so analogously we find that $\# \tau_{d} \lesssim \# \tau$. We conclude that

$$
\# \tau_{p} \cup \tau_{d} \lesssim \# \tau \lesssim \# \tau_{0}+\varepsilon^{-1 /(s+t)}\left[\left(|u|_{\mathcal{A}^{s}}^{1 / s}+c_{f}^{1 / s}\right)^{s}\left(|z|_{\mathcal{A}^{t}}^{1 / t}+c_{g}^{1 / t}\right)^{t}\right]^{1 /(s+t)} .
$$

Finally, we have to bound the cost of the algorithm. At the moment of the first call of GALSOLVE $\left[\tau_{p}, f_{\tau_{p}}, \bar{u}_{\tau_{p}}, \delta_{p}\right]$, we have

$$
\left\|L_{\tau_{p}}^{-1} f_{\tau_{p}}-\bar{u}_{\tau_{p}}\right\|_{E} \leq\left\|f_{\tau_{p}}-f\right\|_{E^{\prime}}+\|f\|_{E^{\prime}} \leq \delta_{p}+\|f\|_{E^{\prime}} \lesssim \delta_{p}
$$

by assumption. We now consider any further calls. From (6.3), $\left\|u-u_{\tau_{0}}\right\|_{E} \leq\|f\|_{E^{\prime}} \lesssim$ $\underline{\delta}_{p}$ by assumption, and (6.5), we have that the currents $\delta_{p}$ and $\sigma_{p}$ at the moment of such a call satisfy $\sigma_{p} \lesssim \delta_{p}$. As a consequence, we have

$$
\begin{aligned}
\left\|L_{\tau_{p}}^{-1} f_{\tau_{p}}-\bar{u}_{\tau_{p}}\right\|_{E} & \leq\left\|\left(L^{-1}-L_{\tau_{p}}^{-1}\right) f_{\tau_{p}}\right\|_{E}+\left\|L^{-1} f_{\tau_{p}}-\bar{u}_{\tau_{p}}\right\|_{E} \leq 2\left\|L^{-1} f_{\tau_{p}}-\bar{u}_{\tau_{p}}\right\|_{E} \\
& \leq 2\left[\left\|f-f_{\tau_{p}}\right\|_{E^{\prime}}+\left\|u-\bar{u}_{\tau_{p}}\right\|_{E}\right] \leq 2 \delta_{p}+2 \sigma_{p} \lesssim \delta_{p} .
\end{aligned}
$$

By the assumption of GALSOLVE being an optimal iterative solver, we conclude that the cost of these calls is $\mathcal{O}\left(\# \tau_{p}\right)$.

The number of arithmetic operations needed for the calls $\mathbf{M A R K}\left[\tau, f_{\tau_{p}}^{1}, \boldsymbol{f}_{\tau_{p}}^{2}, \bar{u}_{\tau_{p}}\right]$, $\tau:=\operatorname{REFINE}\left[\tau_{p}, F_{p}\right]$, and $\left[\tau_{p}, \cdot, \cdot\right]:=\mathbf{R H S}_{f}\left[\tau, \delta_{p}\right]$ are $\mathcal{O}(\# \tau), \mathcal{O}(\# \tau)$, and $\mathcal{O}\left(\# \tau_{p}\right)$,

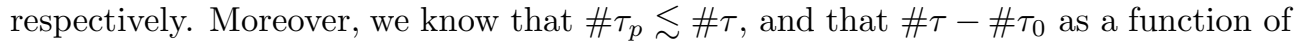
the iteration count is majorized by a linearly increasing sequence with upper bound (6.8). From the assumption that $\underline{\delta}_{p} \underline{\delta}_{d} \lesssim\left\|u-u_{\tau_{0}}\right\|_{E}\left\|z-z_{\tau_{0}}\right\|_{E}+\varepsilon$, the first $\sigma_{p} \sigma_{d} \lesssim$ $\left\|u-u_{\tau_{0}}\right\|_{E}\left\|z-z_{\tau_{0}}\right\|_{E}+\varepsilon$, meaning that after some absolute constant number of iterations, either the current $\tau$ is unequal to $\tau_{0}$ or the algorithm has terminated. Together, above observations show that the total cost is bounded by some absolute multiple of the right-hand side of (6.9).

Remark 6.6. The functions $\bar{u}_{\tau}, \bar{z}_{\tau}$ produced by GOAFEM are not the exact Galerkin approximations, and so $\left\|u-\bar{u}_{\tau}\right\|_{E}\left\|z-\bar{z}_{\tau}\right\|_{E}$ is not necessarily an upper bound for $\left|g(u)-g\left(\bar{u}_{\tau}\right)\right|$. Writing

$g(u)-g\left(\bar{u}_{\tau}\right)=a\left(u-\bar{u}_{\tau}, z\right)=a\left(u-\bar{u}_{\tau}, z-z_{\tau}\right)=a\left(u-\bar{u}_{\tau}, z-\bar{z}_{\tau}\right)-a\left(u-\bar{u}_{\tau}, z_{\tau}-\bar{z}_{\tau}\right)$,

and using the fact that $\left\|u-\bar{u}_{\tau}\right\|_{E} \leq \sigma_{p},\left\|z-\bar{z}_{\tau}\right\|_{E} \leq \sigma_{d},\left\|z_{\tau}-\bar{z}_{\tau}\right\| \leq \delta_{d} \leq(2+$ $\left.C_{1} c_{2}^{-1}\right)^{-1} \sigma_{d}$, and $\sigma_{p} \sigma_{d} \leq \varepsilon$, we end up with $\left|g(u)-g\left(\bar{u}_{\tau}\right)\right| \leq\left[1+\left(2+C_{1} c_{2}^{-1}\right)^{-1}\right] \varepsilon$.

7. Numerical experiments. In this section we will consider the performance of the GOAFEM routine in practice. As many real-world problems require the evaluation of functionals that are unbounded on $H_{0}^{1}(\Omega)$, we will also consider such a problem. As GOAFEM can handle only bounded functionals, we need to do some additional work. Following [BS01], we will apply a so-called extraction functional, a technique that we recall below. An alternative approach would be to apply a regularized functional as suggested in [OR76, BR96]. This approach can be applied more generally since no Green's function is needed. On the other hand, it introduces an additional error that can only be controlled in terms of higher order derivatives of the solution beyond those that are needed for the functional to be well defined.

7.1. Extraction functionals. Let $\tilde{g}$ be some functional defined on the solution $u$ of $(2.1)$, but that is unbounded on $H_{0}^{1}(\Omega)$. With $f$ being the right-hand side of (2.1), we write $\tilde{g}(u)=g(u)+M(f)$, where $g \in H^{-1}(\Omega)$ and $M$ is a functional on 
$f$. Since $u$ and $f$ are related via an invertible operator, this is always possible, even for any $g \in H^{-1}(\Omega)$. Yet, we would like to do this under the additional constraint that $M(f)$ can be computed within any given tolerance at low cost. Basically, this additional condition requires that a Green's function for the differential operator is available.

We consider $\boldsymbol{A}=\mathrm{Id}$, i.e., the Poisson problem, on a two-dimensional domain $\Omega$, and, for some $\bar{x} \in \Omega, \tilde{g}=\tilde{g}_{\bar{x}}$ given by

$$
\tilde{g}_{\bar{x}}(u)=\frac{\partial u}{\partial x_{1}}(\bar{x}),
$$

assuming that $u$ is sufficiently smooth. With $(r, \theta)$ denoting polar coordinates centered at $\bar{x}$, we have $\triangle \frac{\log r}{2 \pi}=\delta_{\bar{x}}$, and so $-\triangle \frac{\cos \theta}{2 \pi r}=\tilde{g}_{\bar{x}}$ in the sense that for any smooth test function $\phi \in \mathcal{D}\left(\mathbb{R}^{2}\right),-\int_{\mathbb{R}^{2}} \frac{\cos \theta}{2 \pi r} \triangle \phi=\tilde{g}_{\bar{x}}(\phi)$. Generally, this formula cannot be applied with $\phi$ replaced by the solution $u$ of (2.1). Indeed, in the general case this function has a nonvanishing normal derivative at the boundary of $\Omega$, and therefore its zero extension is not sufficiently smooth. Therefore, with $w_{0}^{\bar{x}}:=\frac{\cos \theta}{2 \pi r}, w_{1}^{\bar{x}}$ being a sufficiently smooth function equal to $w_{0}^{\bar{x}}$ outside some open $\Sigma \Subset \Omega$ that contains $\bar{x}$, and $w^{\bar{x}}:=w_{0}^{\bar{x}}-w_{1}^{\bar{x}}$ for any $\phi \in \mathcal{D}\left(\mathbb{R}^{2}\right)$, we write

$$
\begin{aligned}
\tilde{g}_{\bar{x}}(\phi) & =-\int_{\mathbb{R}^{2}} w_{1}^{\bar{x}} \triangle \phi-\int_{\mathbb{R}^{2}} w^{\bar{x}} \triangle \phi \\
& =\int_{\mathbb{R}^{2}} \triangle\left(-w_{1}^{\bar{x}}\right) \phi+\int_{\Omega} w^{\bar{x}}(-\triangle \phi) \\
& =: g_{\bar{x}}(\phi)+M_{\bar{x}}(-\triangle \phi) .
\end{aligned}
$$

Clearly, $g_{\bar{x}}$ extends to a bounded functional on $L_{1}\left(\mathbb{R}^{2}\right)$, with $g_{\bar{x}}(v)=\int_{\Omega} \triangle\left(-w_{1}^{\bar{x}}\right) v$ when $\operatorname{supp} v \subset \Omega$. In particular, $g_{\bar{x}}$ is bounded on $H_{0}^{1}(\Omega)$, which enables us to use GOAFEM to evaluate it. Moreover, since supp $w^{\bar{x}} \Subset \Omega$, under some mild conditions the above reformulation can be shown to be applicable to $u$. The details are as follows.

Proposition 7.1. If

(a) $f \in L_{2}(\Omega)$,

(b) $u$ is continuously differentiable at $\bar{x}$, and then

(c) in a neighborhood of $\bar{x}, f$ is in $L^{p}$ for some $p>2$,

$$
\tilde{g}_{\bar{x}}(u)=g_{\bar{x}}(u)+M_{\bar{x}}(f) .
$$

Proof. Let $B(\bar{x} ; \varepsilon)$ be the ball centered at $\bar{x}$ with radius $\varepsilon$, and small enough such that $B(\bar{x} ; \varepsilon) \Subset \Omega$. Since $u, w^{\bar{x}} \in H^{1}(\Omega \backslash B(\bar{x} ; \varepsilon)), \triangle u \in L_{2}(\Omega \backslash B(\bar{x} ; \varepsilon))$ by (a), $\triangle w^{\bar{x}} \in L_{2}(\Omega \backslash B(\bar{x} ; \varepsilon))$, and supp $w^{\bar{x}} \Subset \Omega$, integration by parts shows that

$$
\int_{\partial B(\bar{x} ; \varepsilon)} w^{\bar{x}} \frac{\partial u}{\partial \mathbf{n}}-u \frac{\partial w^{\bar{x}}}{\partial \mathbf{n}}=\int_{\Omega \backslash B(\bar{x} ; \varepsilon)} u \triangle w^{\bar{x}}-w^{\bar{x}} \triangle u
$$

where $\mathbf{n}$ is the outward pointing normal of $\partial B(\bar{x} ; \varepsilon)$.

We have $\lim _{\varepsilon \downarrow 0} \int_{\Omega \backslash B(\bar{x} ; \varepsilon)} u \triangle w^{\bar{x}}=-\lim _{\varepsilon \downarrow 0} \int_{\Omega \backslash B(\bar{x} ; \varepsilon)} u \triangle w_{1}^{\bar{x}}=g_{\bar{x}}(u)$.

Since $\left|\int_{B(\bar{x} ; \varepsilon)} w_{0}^{\bar{x}} f\right| \leq\|f\|_{L_{p}(B(\bar{x} ; \varepsilon))}\left\|w_{0}^{\bar{x}}\right\|_{L_{q}(B(\bar{x} ; \varepsilon))}\left(\frac{1}{p}+\frac{1}{q}=1\right)$, and furthermore $\left\|w_{0}^{\bar{x}}\right\|_{L_{q}(B(\bar{x} ; \varepsilon))}=\left[\int_{0}^{\varepsilon} \int_{0}^{2 \pi}\left|\frac{\cos \theta}{2 \pi r}\right|{ }^{q} r\right]^{1 / q} \rightarrow 0$ when $\varepsilon \downarrow 0$ and $q<2$, from (c) we conclude that $-\lim _{\varepsilon \downarrow 0} \int_{\Omega \backslash B(\bar{x} ; \varepsilon)} w^{\bar{x}} \triangle u=\int_{\Omega} w^{\bar{x}} f=M_{\bar{x}}(f)$. 
The contributions of $w_{1}^{\bar{x}}$ to the left-hand side of (7.1) vanish when $\varepsilon \downarrow 0$.

From $\int_{\partial B(\bar{x} ; \varepsilon)} w_{0}^{\bar{x}} \frac{\partial u}{\partial \mathbf{n}}=\int_{0}^{2 \pi}\left(\cos \theta \frac{\partial u}{\partial x_{1}}+\sin \theta \frac{\partial u}{\partial x_{2}}\right) \frac{\cos \theta}{2 \pi \varepsilon} \varepsilon d \theta$ and (b), we infer that $\lim _{\varepsilon \downarrow 0} \int_{\partial B(\bar{x} ; \varepsilon)} w_{0}^{\bar{x}} \frac{\partial u}{\partial \mathbf{n}}=\frac{1}{2} \frac{\partial u}{\partial x_{1}}(\bar{x})$.

From

$$
\begin{aligned}
\int_{\partial B(\bar{x} ; \varepsilon)} u \frac{\partial w_{0}^{\bar{x}}}{\partial \mathbf{n}} & =\frac{-1}{2 \pi \varepsilon} \int_{0}^{2 \pi} \cos \theta u d \theta=\frac{1}{2 \pi \varepsilon} \int_{0}^{2 \pi} \sin \theta \frac{\partial u}{\partial \theta} d \theta \\
& =\frac{1}{2 \pi} \int_{0}^{2 \pi} \sin \theta\left(-\sin \theta \frac{\partial u}{\partial x_{1}}+\cos \theta \frac{\partial u}{\partial x_{2}}\right) d \theta
\end{aligned}
$$

and (b), we infer that $-\lim _{\varepsilon \downarrow 0} \int_{\partial B(\bar{x} ; \varepsilon)} u^{\bar{x}} \frac{\partial w_{0}^{\bar{x}}}{\partial \mathbf{n}}=\frac{1}{2} \frac{\partial u}{\partial x_{1}}(\bar{x})$. Together, the above observations give the proof.

7.2. Implementation. The implementation of the GOAFEM routine is essentially as described above, with the sole difference that we did not approximate the right-hand sides for setting up the Galerkin systems and computing the a posteriori error estimators, but instead used quadrature directly. This was possible, and in view of Remark 6.2 reasonable, because in our experiments either the right-hand sides are very smooth or they are already in $\mathbb{V}_{\tau_{0}}^{*}+\operatorname{div}\left[\mathbb{V}_{\tau_{0}}^{*}\right]^{n}$.

For all experiments, we used $p=2$, i.e., quadratic Lagrange elements.

The GALSOLVE routine we use solves the linear systems with the conjugate gradient method using the well-known Bramble-Pasciak-Xu preconditioner.

All routines were implemented in Common Lisp and run using the SBCL compiler and run-time environment. This allowed for a short development time and wellinstrumented code. With regards to efficiency, the only effort made in that direction consisted in making sure that the asymptotics were correct. While an efficient implementation would be possible with moderate effort (see [Neu03]), for our purposes convenience and correctness were the most important considerations.

For the experiment in which we use the extraction functional for the partial derivative at a point introduced above, we also have to solve a quadrature problem. For this we used the adaptive cubature routine Cuhre [BEG91] as implemented in the Cuba cubature package [Hah05].

7.3. Experiments. To test GOAFEM, we chose two distinct situations. For the first example, we want to compute a partial derivative at a point of a function given as the solution of a Poisson problem, thus illustrating the applicability of our method to this situation.

In our second example, we consider a problem in which the singularities of the solutions to the primal and dual problems are spatially separated.

Example 7.2. Let $\Omega=(0,1)^{2}$. We consider problem (2.1), choosing the righthand side $f=1$ (i.e., $f(v)=\int_{\Omega} v d x$ ). We will test the performance of GOAFEM on the task of computing

$$
\frac{\partial u}{\partial x_{1}}(\bar{x})
$$

with $\bar{x}=\left(\frac{\pi}{7}, \frac{49}{100}\right)$. The initial partition is as indicated in Figure 7.1, with $\left(\frac{1}{2}, \frac{1}{2}\right)$ being the newest vertex of all 4 triangles.

Following the discussion from subsection 7.1, we take $w_{1}^{\bar{x}}=\psi w_{0}^{\bar{x}}$, and thus $w^{\bar{x}}=$ $(1-\psi) w_{0}^{\bar{x}}$, with $\psi$ being a sufficiently smooth function, 1 outside some neighborhood 


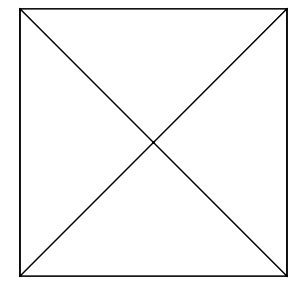

FIG. 7.1. Initial partition $\tau_{0}$ corresponding to Example 7.2.

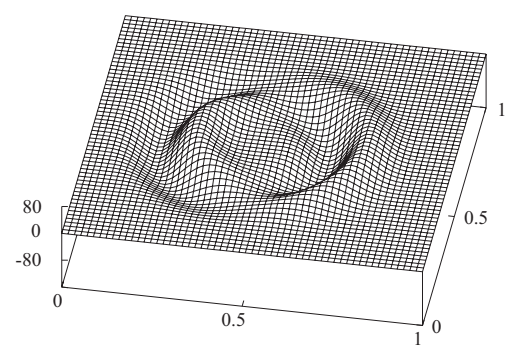

Fig. 7.2. Right-hand side of the dual problem corresponding to Example 7.2.

of $\bar{x}$ inside $\Omega$, and 0 on some smaller neighborhood of $\bar{x}$. Proposition 7.1 shows that $\frac{\partial u}{\partial x_{1}}(\bar{x})=\int_{\Omega} u \triangle\left(-\psi w_{0}^{\bar{x}}\right)+\int_{\Omega}(1-\psi) w_{0}^{\bar{x}} f$. Writing $(\theta, r)$ for the polar coordinates around $\bar{x}$, we chose

$$
\psi(\theta, r):=\int_{0}^{r} \psi^{*}(s) d s / \int_{0}^{\infty} \psi^{*}(s) d s,
$$

with $\psi^{*}$ a spline function of order 6 , with support [0.1, 0.45].

We evaluated $\int_{\Omega}(1-\psi) w_{0}^{\bar{x}} f$ using the adaptive quadrature routine Cuhre. To obtain precision of $10^{-12}$ it needed 216515 integrand evaluations. On current off-theshelf hardware, it takes only a few seconds.

To approximate $\int_{\Omega} u \triangle\left(-\psi w_{0}^{\bar{x}}\right)$ we used GOAFEM. Since the right-hand sides 1 and $\triangle\left(-\psi w_{0}^{\bar{x}}\right)$ of primal and dual problems are smooth, their solutions are in $\mathcal{A}^{p / n}=\mathcal{A}^{1}$, so that the error in the functional is $\mathcal{O}\left(\left[\# \tau-\# \tau_{0}\right]^{-2}\right)$. We compared the results with those obtained with the corresponding non-goal-oriented adaptive finite element routine AFEM for minimizing the error in energy norm, which is obtained by applying refinements always because of the markings at primal side.

The solutions of the primal and dual problems are in $H^{3-\varepsilon}(\Omega)$ for any $\varepsilon>0$, but, because the right-hand sides do not vanish at the corners, they are not in $H^{3}(\Omega)$. Recalling that we use quadratic elements, as a consequence (fully) optimal convergence rates with respect to \|\|$_{E}$ are not obtained using uniform refinements. On the other hand, since the (weak) singularities in the primal and dual solutions are solely caused by the shape of the domain, the same local refinements near the corners are appropriate for both primal and dual problem. Therefore, in view of (1.1), we may expect that also with AFEM the error in the functional is $\mathcal{O}\left(\left[\# \tau-\# \tau_{0}\right]^{-2}\right)$. On the other hand, since quantitatively the right-hand side, and so the solution of the dual problem, are not that smooth (see Figure 7.2), we may hope that the application of GOAFEM yields quantitatively better results.

In Figure 7.3, we show errors in $\int_{\Omega} u \triangle\left(-\psi w_{0}^{\bar{x}}\right)$ as a function of $\# \tau-\# \tau_{0}$. The re- 


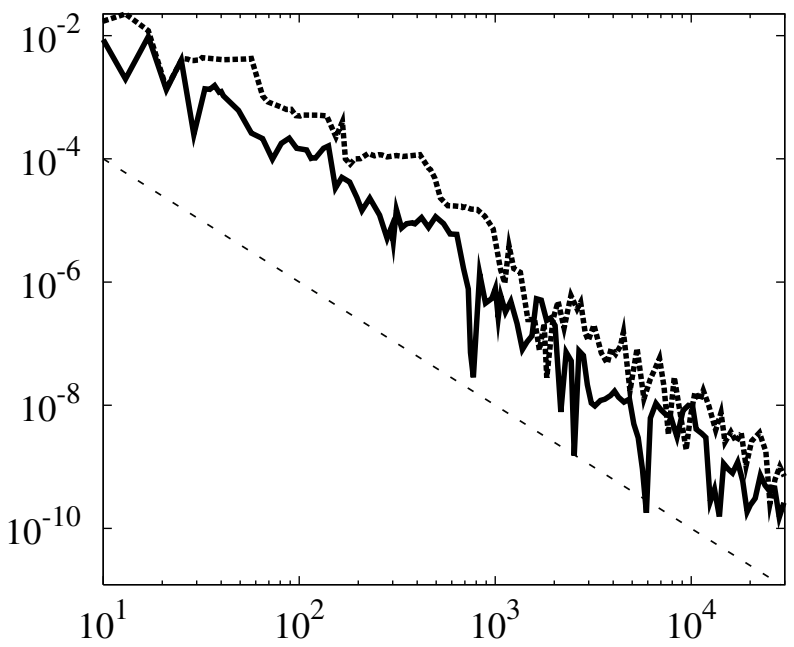

FIG. 7.3. Error in the functional vs. \# $\tau-\# \tau_{0}$ using GOAFEM (solid) and AFEM (dashed) corresponding to Example 7.2 , and a curve $C\left[\# \tau-\# \tau_{0}\right]^{-2}$.
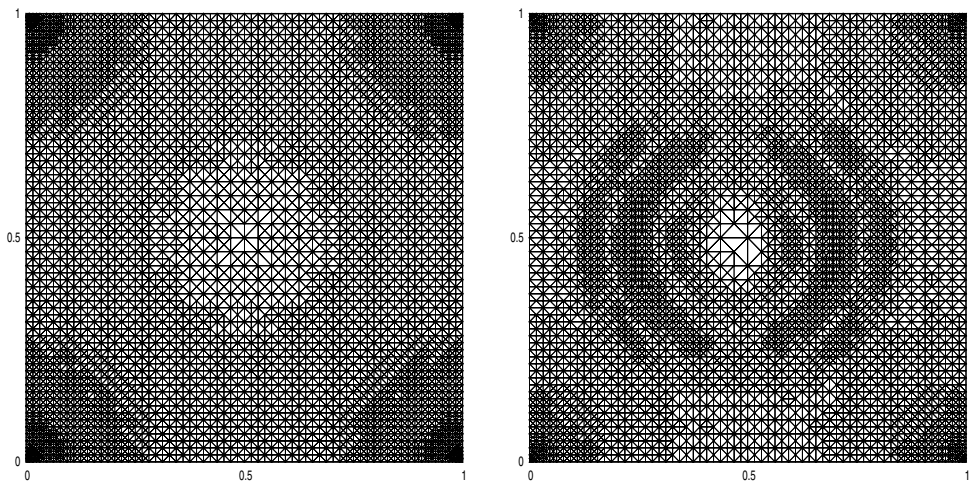

Fig. 7.4. Partitions produced by AFEM and GOAFEM with nearly equal number of triangles for Example 7.2.

sults confirm that for both GOAFEM and AFEM, these errors are $\mathcal{O}\left(\left[\# \tau-\# \tau_{0}\right]^{-2}\right)$, where on average for GOAFEM the errors are smaller. In Figure 7.4, we show partitions produced by GOAFEM and AFEM. With AFEM local refinements are made only towards the corners, whereas with GOAFEM additional local refinements are made in areas where quantitatively the dual solution is nonsmooth due to oscillations in its right-hand side.

Example 7.3. As in Example 7.2, we consider Poisson's problem on the unit square. We now take as initial partition the one that is obtained from the partition from Figure 7.1 by 2 uniform refinements. We define the right-hand sides $f$ and $g$ of primal and dual problems by

$$
f(v)=-\int_{T_{f}} \frac{\partial v}{\partial x_{1}}, \quad g(v)=-\int_{T_{g}} \frac{\partial v}{\partial x_{1}},
$$




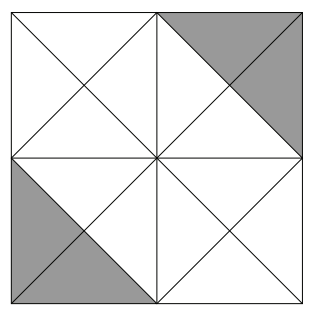

FIG. 7.5. Initial partition $\tau_{0}$ corresponding to Example 7.3, and $T_{f}$ (left bottom), $T_{g}$ (right top).

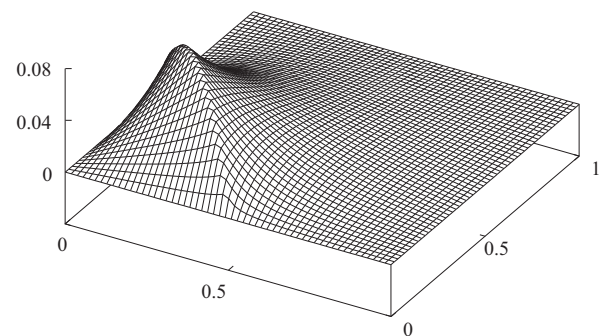

FIG. 7.6. Primal solution corresponding to Example 7.3.

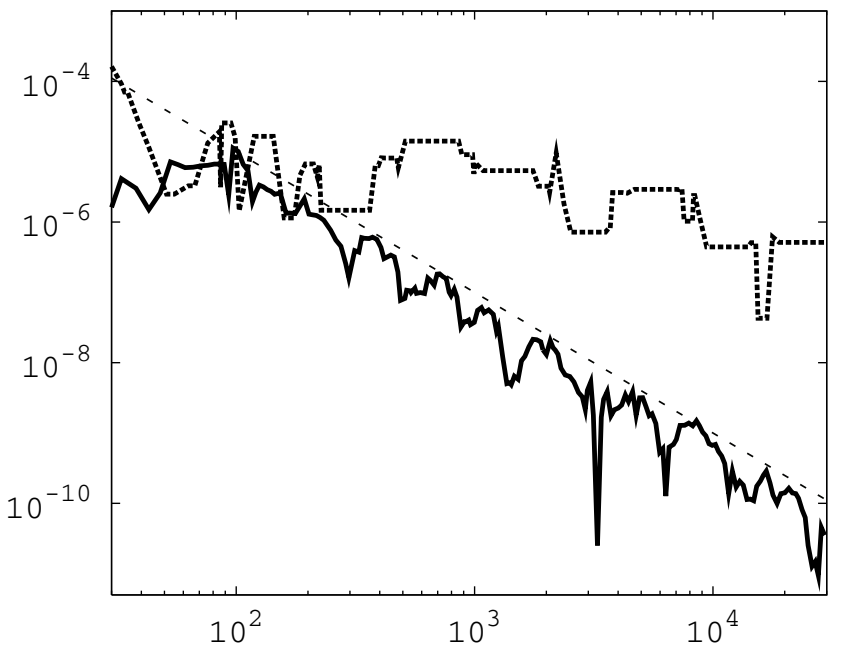

FIG. 7.7. Error in the functional vs. \# $\tau-\# \tau_{0}$ using GOAFEM (solid) and AFEM (dashed) corresponding to Example 7.3 , and a curve $C\left[\# \tau-\# \tau_{0}\right]^{-2}$.

where $T_{f}$ and $T_{g}$ are the simplices $\left\{(0,0),\left(\frac{1}{2}, 0\right),\left(0, \frac{1}{2}\right)\right\}$ and $\left\{(1,1),\left(\frac{1}{2}, 1\right),\left(1, \frac{1}{2}\right)\right\}$, respectively; see Figure 7.5. That is, with $\chi_{f}$ being the characteristic function of $T_{f}$, $f=\operatorname{div}\left[\begin{array}{ll}\chi_{f} & 0\end{array}\right]^{T}$. So in view of (4.3), here we write $f$ as $f^{1}+\operatorname{div} f^{2}$ with vanishing $f^{1}$, and benefit from the fact that $f^{2} \in\left[V_{\tau_{0}}^{*}\right]^{2}$. Similarly for $g$.

The primal solution has a singularity along the line connecting the points $\left(\frac{1}{2}, 0\right)$ and $\left(0, \frac{1}{2}\right)$ (see Figure 7.6), and similarly the dual solution has one along the line connecting $\left(1, \frac{1}{2}\right)$ and $\left(\frac{1}{2}, 1\right)$. Since the non-goal-oriented adaptive finite element routine AFEM does not see the latter singularity, it behaves much worse than GOAFEM, as seen in Figure 7.7. For GOAFEM we observe an error $\mathcal{O}\left(\left[\# \tau-\# \tau_{0}\right]^{-2}\right)$, which, since 

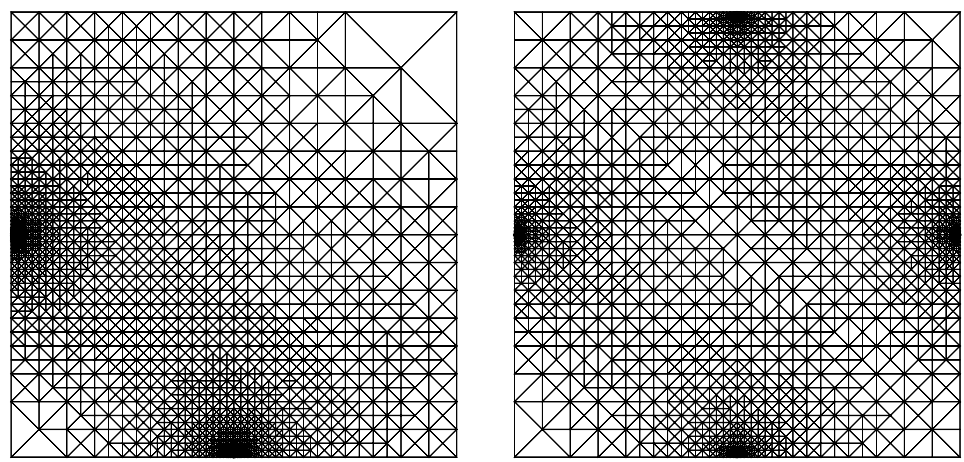

FIG. 7.8. Partitions produced by AFEM and GOAFEM with nearly equal number of triangles for Example 7.3.

$p / n=1$, is equal to the best possible rate predicted by Theorem 6.4. In Figure 7.8, we show partitions produced by AFEM and GOAFEM, respectively.

\section{REFERENCES}

[AO00] M. Ainsworth And J. T. Oden, A Posteriori Error Estimation in Finite Element Analysis, Pure Appl. Math., Wiley-Interscience, New York, 2000.

[BR96] R. BECKER AND R. RANNACHER, A feed-back approach to error control in finite element methods: Basic analysis and examples, East-West J. Numer. Math., 4 (1996), pp. 237-264

[BDD04] P. Binev, W. Dahmen, and R. DeVore, Adaptive finite element methods with convergence rates, Numer. Math., 97 (2004), pp. 219-268.

[BDdP02] P. Binev, W. Dahmen, R. DeVore, and P. Petruchev, Approximation classes for adaptive methods, Serdica Math. J., 28 (2002), pp. 391-416.

[BEG91] J. Bernsten, T. O. Espelid, AND A. Genz, An adaptive algorithm for the approximate calculation of multiple integrals, ACM Trans. Math. Software, 17 (1991), pp. 437451.

[BMn02] E. BÄNSch, P. Morin, And R. H. Nochetto, An adaptive Uzawa FEM for the Stokes problem: Convergence without the inf-sup condition, SIAM J. Numer. Anal., 40 (2002), pp. 1207-1229.

[BR03] W. Bangerth and R. Rannacher, Adaptive Finite Element Methods for Differential Equations, Lectures Math. ETH Zürich, Birkhäuser Verlag, Basel, 2003.

[BS01] I. BabušKa And T. Strouboulis, The Finite Element Method and Its Reliability, Numer. Math. Sci. Comput., The Clarendon Press, Oxford University Press, New York, 2001.

[DKV06] W. Dahmen, A. Kunoth, And J. Vorloeper, Convergence of adaptive wavelet methods for goal-oriented error estimation, in Numerical Mathematics and Advanced Applications, Springer-Verlag, Berlin, 2006, pp. 39-61.

[Dör96] W. Dörfler, A convergent adaptive algorithm for Poisson's equation, SIAM J. Numer. Anal., 33 (1996), pp. 1106-1124.

[Hah05] T. HAHn, Cuba-a library for multidimensional numerical integration, Comput. Phys. Comm., 168 (2005), pp. 78-95.

[KS08] Y. Kondratyuk and R. P. Stevenson, An optimal adaptive finite element method for the Stokes problem, SIAM J. Numer. Anal., 46 (2008), pp. 747-775.

[Mau95] J. M. MAUBACH, Local bisection refinement for $n$-simplicial grids generated by reflection, SIAM J. Sci. Comput., 16 (1994), pp. 210-227.

[MNS00] P. Morin, R. Nochetto, AND K. Siebert, Data oscillation and convergence of adaptive FEM, SIAM J. Numer. Anal., 38 (2000), pp. 466-488.

[MS08] M. Mommer And R. P. Stevenson, A Goal-Oriented Adaptive Finite Element Method with Convergence Rates-Extended Version, preprint, Korteweg-de Vries Institute for Mathematics, University of Amsterdam, 2008; extended preprint version of current work on http://staff.science.uva.nl/ rstevens/publ.html. 
[MvSST06] K.-S. Moon, E. von Schwerin, A. Szepessy, And R. Tempone, Convergence rates for an adaptive dual weighted residual finite element algorithm, BIT, 46 (2006), pp. 367-407.

[Neu03] N. Neuss, On using Common Lisp in scientific computing, in Challenges in Scientific Computing-CISC 2002, Springer-Verlag, Berlin, 2003, pp. 237-245.

[OR76] J. T. Oden AND J. N. RedDy, An Introduction to the Mathematical Theory of Finite Elements, Pure Appl. Math., Wiley-Interscience, New York, 1976.

[Ste05] R. P. Stevenson, An optimal adaptive finite element method, SIAM J. Numer. Anal., 42 (2005), pp. 2188-2217.

[Ste07] R. P. Stevenson, Optimality of a standard adaptive finite element method, Found. Comput. Math., 7 (2007), pp. 245-269.

[Ste08] R. P. Stevenson, The completion of locally refined simplicial partitions created by bisection, Math. Comp., 77 (2008), pp. 227-241.

[Tra97] C. T. Traxler, An algorithm for adaptive mesh refinement in $n$ dimensions, Computing, 59 (1997), pp. 115-137.

[Ver96] R. Verfürth, A Review of A Posteriori Error Estimation and Adaptive MeshRefinement Techniques, Wiley-Teubner, Chichester, UK, 1996. 